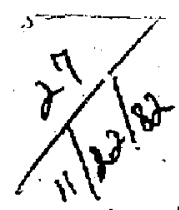

(V)

\subsection{4}

d

\section{FIELD EVALUATION OF PROTOTYPE ELECTROFIBROUS FILTERS}

4. D. Kuhl, H. Bergman, A. H. Biermarin and

B. Y. Lum

September 30, 1982

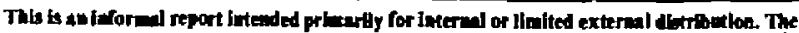

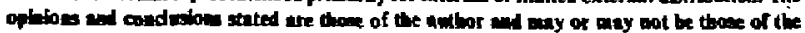
Lithatery.

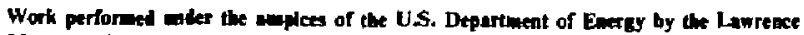
Livereare Lutotary inder Contract W-7405-Eng-49. 


\author{
Field Evaluation of Prototype Electrofibrous filters* \\ W. D. Kuhl, W. Bergman, A. H. Biermann and B. Y. Lum \\ Lawrence Livermore National Laboratory \\ Livermore, California 94550
}

\title{
ABSTRACT
}

New prototype electrofibrous filters were designed, built and evaluated in laboratory tests and in field installations. Two prototypes were designed for use in nuclear ventilation ducts as prefilters to HEPA filters. One protot_je is designed to be a permanent component of the ventilation system while the other is a disposable unit. The dispasable electrofibrous prefilter was installed in the exhaust stream of a glove box in which barrels of uranium turnings are burned. Preliminary tests show the disposable prefilter is effectively prolonging the HEPA filter life. An earlier prototype of the rolling prefilter was upgraded to meet the increased requirements for installation in a nuclear facility. This upgraded prototype wac evaluated in the fire test facility at LLNL and shown to be effective is protecting HEPA fijters from plugging under the most severe smoke conditions. The last prototype described in this report is a recirculating air filter. After demonstrating a high performance in laboratory tests the unit was shioped to Savannah River where it is awaiting installation in a Pu fuel fabrication facility. An analysis of the particulate problem in savannah River indicates that four recirculating air filters will save $\$ 172,000$ per year in maintenance costs. 


\section{Introduction}

This report describes the field evaluation of three prototype electrofibrous filters: a stationary prefilter, a rolling prefilter and a recirculating air filter. Although the designs and applications of these prototypes vary considerably, they are all based on the same principle of electrofibrous filters. The designs of these prototypes were obtained by optimizing a number of parameters that determine the performance of the electrofibrous filter. We have conducted extensive theoretical and laboratory investigations to provide the foundation for designing electrofibrous filters for a given application. (1-4) The design parameters and criteria for building electrofibrous filters have been presented in another report. (5)

Before discussing the specific prototypes presented in this report we wit? review the fundamental design on which these electrofibrous filters are based and the two characteristic features of these filters. Figu'e i shows the bas ic components of the electrofibrous filter. A fibrous filter medium is sandwiched between two perforated electrodes separated by a spacer. By applying high voltage to one electrode and grounding the other, an electric

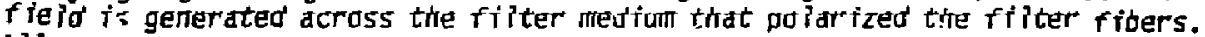
All of thie prototype filters described in this report have the same basic components.

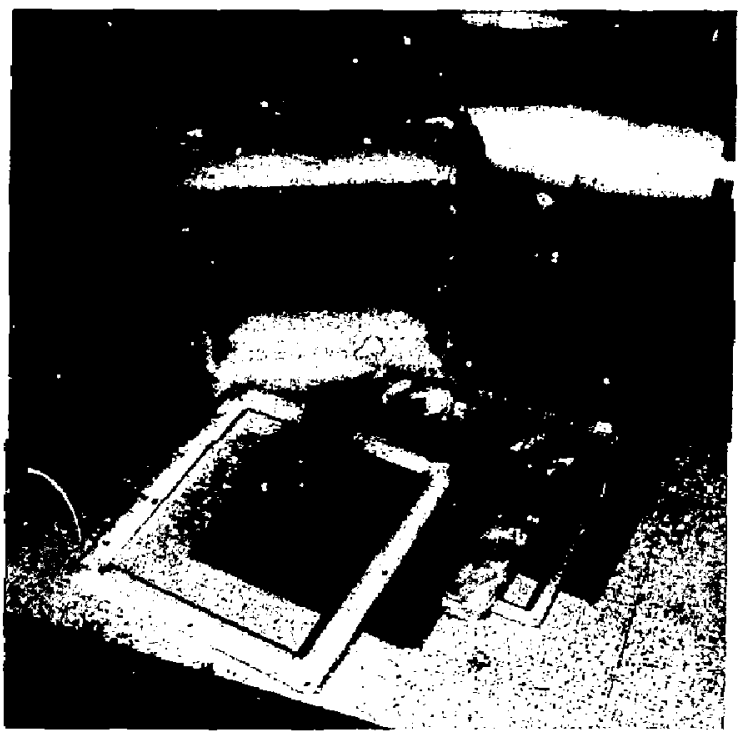

Figure 1. Components of electrofibrous filter. 
The two characteristic features that result from this basic design are (1) a righer filter efficiency and (2) on increased particle laading when compared to the unelectrified filter medium. Figure 2 shows that electrifying the filter medium will increase the filter efficiency over all particle sizes. The increase is especially pronounced over the particle size range in which the unelectrified medium shows a minimum efficiency. The increased particle loading that occurs when a filter medium is electrified is shown in Fig. 3. We have previously shown that the increased particle loading is due to changes in the particle deposits caused by the electrical forces. $(1,3,4,5)$

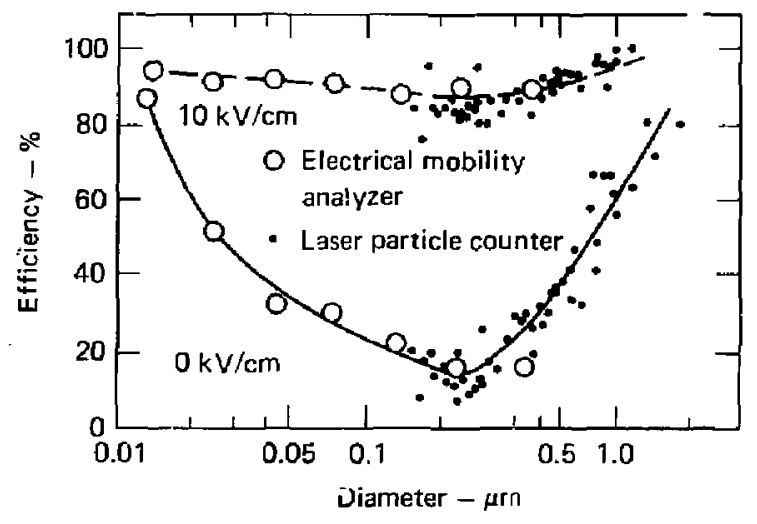

Figure 2. Filter efficiency as a function of particle size with and without an electric field. 


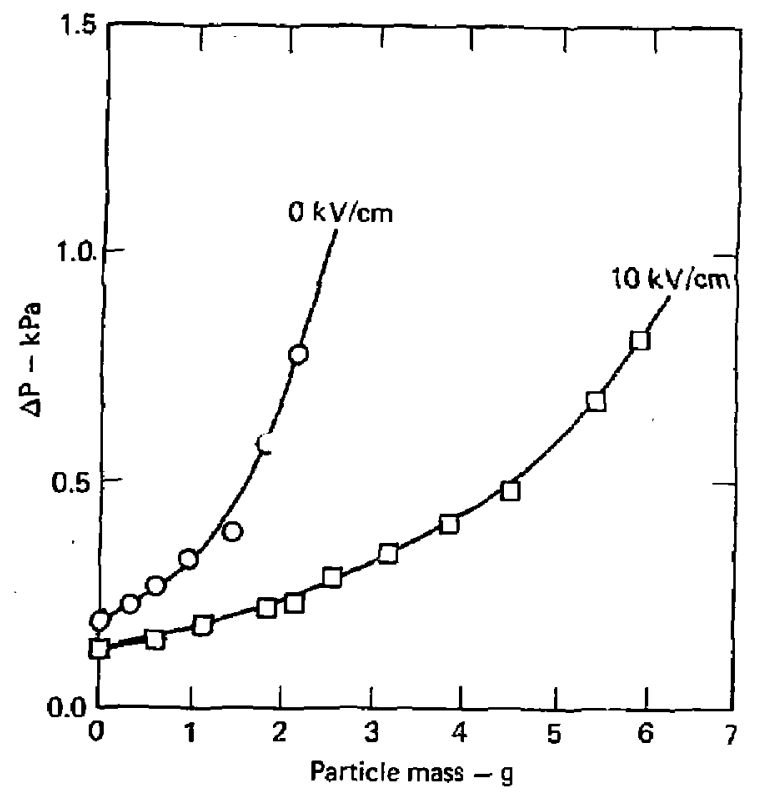

figure 3. Electrifying a filter medium increases its particle loading capacity. 
II. Evaluation of Stationary Electrofibrous Prefilter in LLNL's Uranium Burn Bax

A. Introduction

We haye designed two prototype electrofibrous filters for use in nuclear ventilation systems as prefilters for HEPA filters. Both prototypes have the overall dimensions of a standard HEPA filter $\left(2^{\prime} \times 2^{\prime} \times 1^{\prime}\right)$ and have the filter media pleated to increase the filtering area. These prefilters are designed to significantly extend the life of the downstreari HEPA $f$ ilters and reduce the overail costs and radicactive waste of the prefilter-HEPA filter system.

However, since the cost for filter replacement is a major fraction of the overall filtration cost in a nuclear facility, the use of a prefilter, whether conventional design or electrified, is cost effective only when the replacement cost for the prefilter is much lower than that for the HEPA filter. If the replacement of a prefilter requires a comparable amount of work as replacing a HEPA filter, the use of a prefilter will not be cost effective.

The housing in which the prefilter is installed must have provisions for easily replacing or cleaning the prefilter. Unfortunately, filter housings for prefilters have histarically had the same basic design as used for HEPA filters. Therefore, replacing a prefilter in these housings required about the sane effort as replacing a HEPA filter. It is, therefore, not surprising that prefilters have not been widely used in nuclear ventilation systems.

\section{B. Stationary Electrofibrous Prefilter Designs}

We recognized the importance of a properly designed prefilter housing and have designed our electrofibrous prefilters and $f i l$ ter housing as an integrated prefiftration system having minimal maintenance. We have designed and built two different models that represent the two extremes of the basic design in which electrified media is pleated to increase the filtering area.

Figure 4 shows one of our stationary prefilter models mounted inside a ventilation housing that was designed for minimizing prefiltration maintenance. To increase the flexibility of our evaluation program, we have built two separate filter housings into a single unit and have joined the inlet and exhaust openings into a common duct. This ventilation sys tem permits us considerable flexibility in our field evaluations. We can simultaneously evaluate two prefilters or a prefilter and HEPA. filter under the same conditions and divert the air flow from one chamber to the other. The primary features of the ventilation housing in Fig. 4 are the glove ports and entry-exit ports which permit prefilter replacement or cleaning without disrupting the ventilation flow. We have essentially built a glove box around the prefilter to allow in-situ maintenance on the prefilter. 


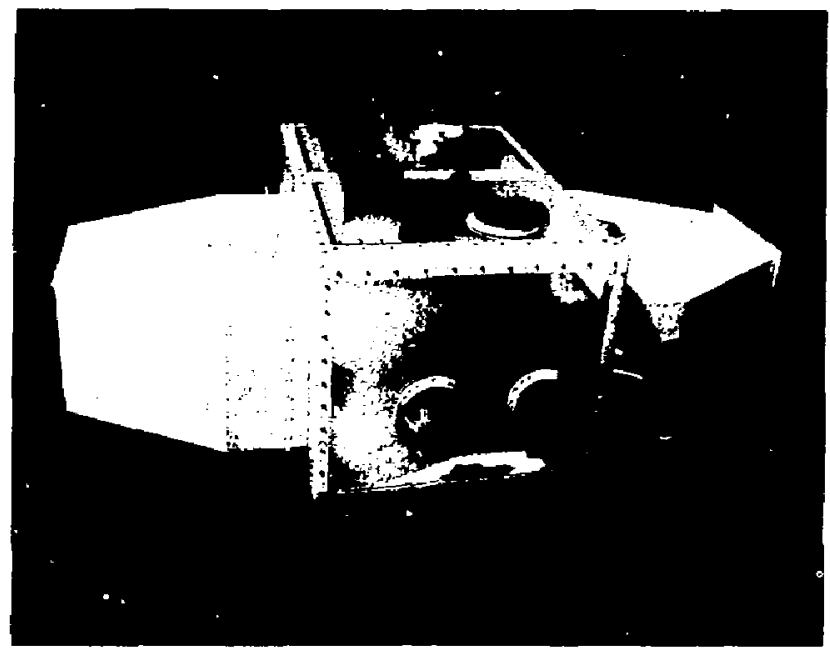

Figure 4. Stationary electrofibrous prefilter mounted inside a low maintenance ventilation housing.

One model of our stationary electrofibrous prefilter is desigred to be a permanent component of the ventilation system. It has a rigid stainless steel frame and durable high voltage and ground electrodes. This prefilter design, shown in Fig. 5, uses an inexpensive filter medium that is inserted into the filter unit and removed when it becomes plugged. The filter medium is sandwiched between high voltage and ground electrodes that are pleated to increase the filtering surface area. The front ground electrodes can be pulled out of the filter unit to provide access to the filter medium. Our previous design that was described in reference 5 required an operator to support the front electrode as it was pulled out of the filter unit. That design proved to be impractical because the electrode was heavy and bulky. thereby making the filter medium replacement an awkward and complicated operation. We have significantly improved the filter medium replacement operation by mounting the front electrode on slides that support the electrode as it is pulled out of the filter unit. We have designated this stationary e lectrofibrous prefilter as our permanent filter model. 


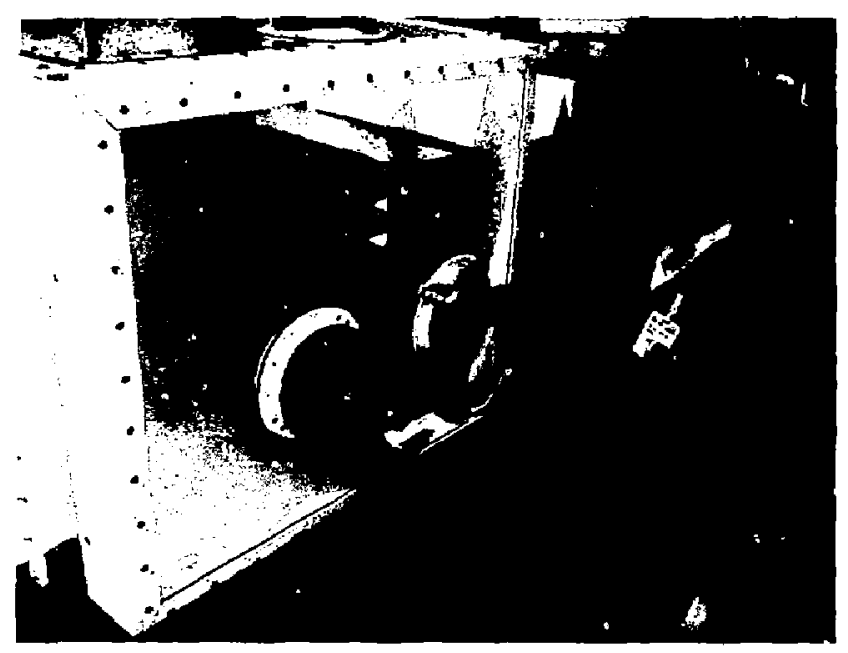

Figure 5. Stationary electrofibrous prefilter designed as a permanent component of the ventilation system.

The operation of replacing the filter medium in our permanent filter model is illustrated in the following sequence of photographs. Figures 6 and 7 show an operator pulling the front electrode away from the filter unit until it clears the filter unit. The front electrode is then rotated in Fig. 8 to permit the filter medium to be slipped over the pleated electrode as shown in Fig. 9. Note that the filter medium is preformed to fit over the front e lectrode. After the filter medium is slipped over the front electrode, the front electrode is rotated back into its normal position and pushed into the main filter unit as shown in Fig. 10. Filter media is passed into the filter hous ing through the exit-entry ports as shown in Figs. 11 and 12. The primary operating feature of the permanent filter design is the use of an inexpensive, disposable filter medium in a permanent electrofibrous filter unit. 


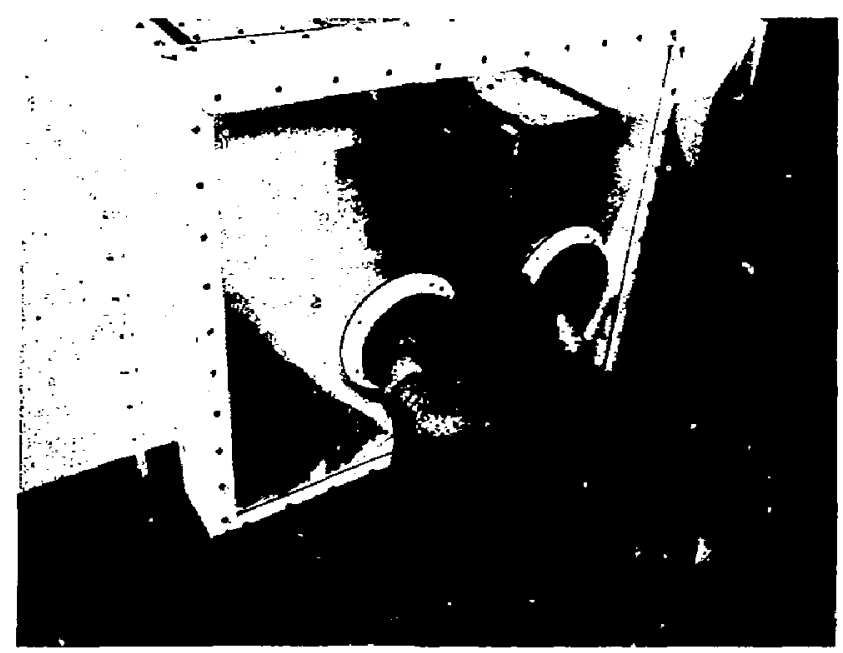

Figure 6. Operator pulling front electrode out of filter unit.

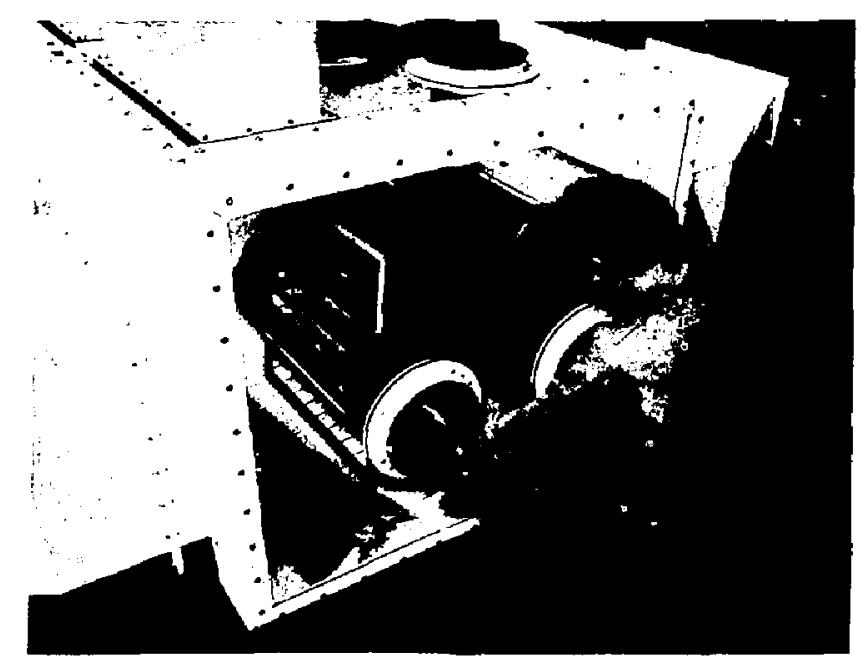

Figure 7. Front electrode pulled out of filter unit. 


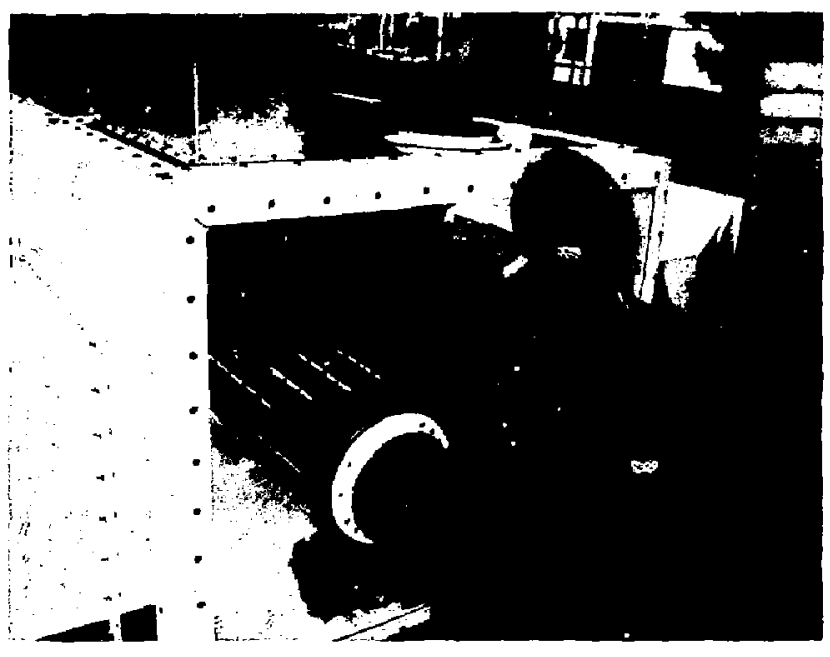

Figure 8 . Front electrode rotated for inserting filter medium.

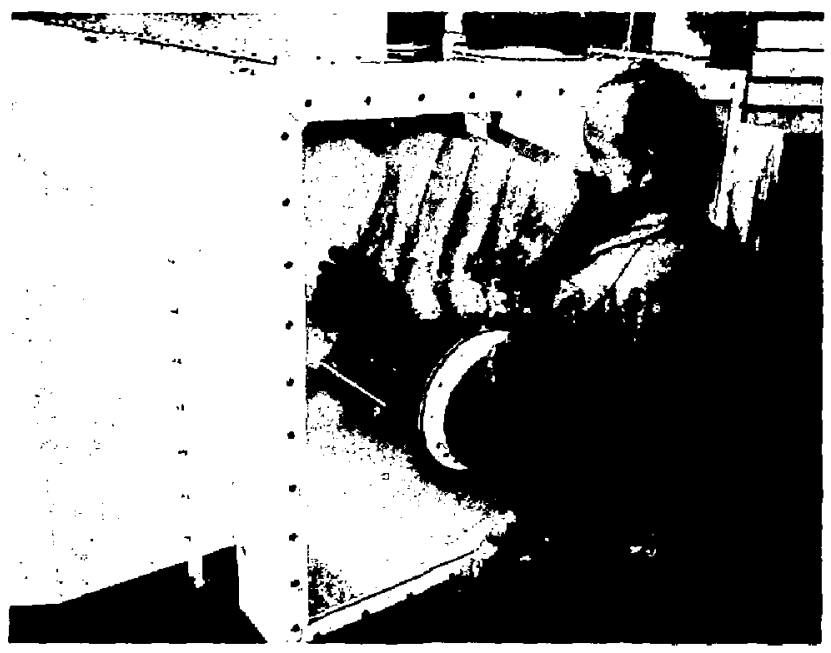

Figure 9. Operator inserting filter medium over front electrode. 


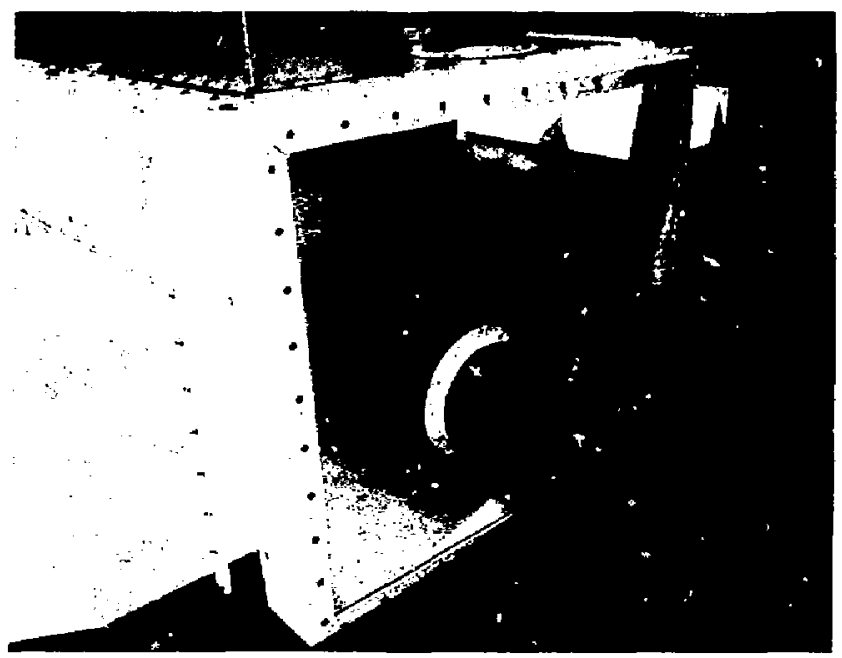

Figure 10. Front electrode witil fresh filter medium being pushed into the filter unit.

The second model of our stationary electrofibrous prefilter design is a disposable filter unit that consists of a sealing edge, a pleated filter aredium and high voltage and ground electrodes as an integrated unit. Figures 13 and 14 show the inlet and exit sides of the disposable electrofibrous prefilter respectively. The high voltage and ground electrodes are glued on the pleated filter medium and the filter medium glued to the square sealing frame. The electrodes were cut from a flexible stainless stee] screen. TC prevent short circuiting between the high voltage and ground electrodes at the sewn edges of the filter media, we used rectangular screen segments rather than a continuous strip. The screen segnents were cut to maintain a 1.5 inch border around the perimeter of each pleat. The ground electrode segments shown in Fig. 14 were electrically connected to the sealing frame by means of a flexible cable. Figure 13 shows an electrode bus that connects the high voltage electrodes to a common lead. "ite that high voltage is applied to the interior electrodes to prevent short circuiting with the ventilation duct in case of accidental misuse or improper installation. 


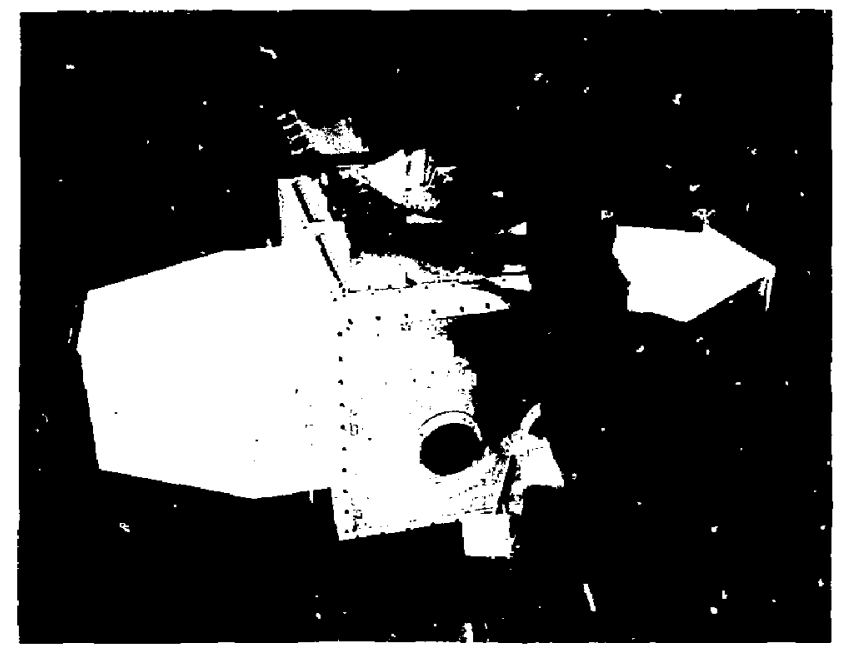

Figure 11. Operator inserting a fresh filter medium into the entry-exit port.

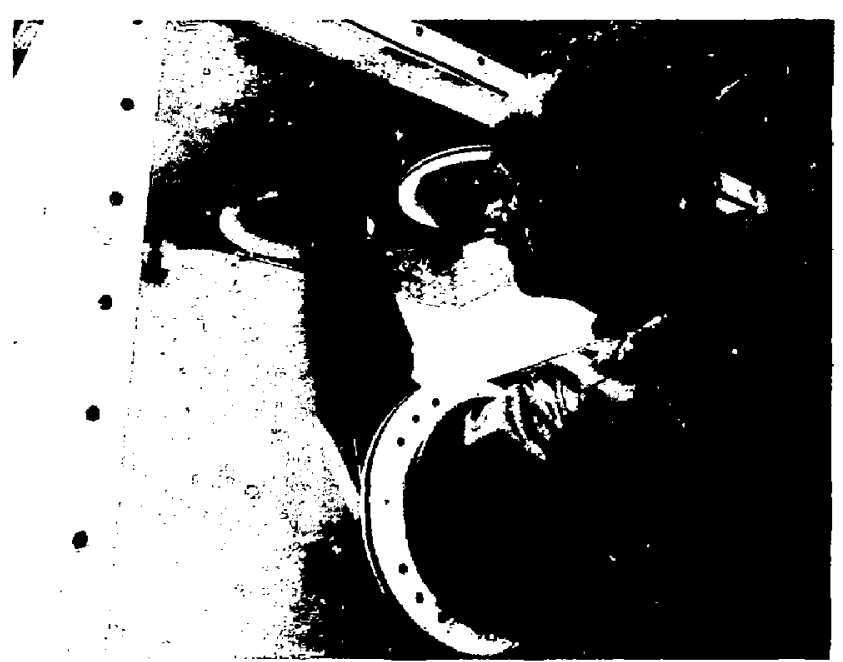

Figure 12. 0,erator opening the entry-exit port to bring a fresh filter medium into the ventilation housing. 


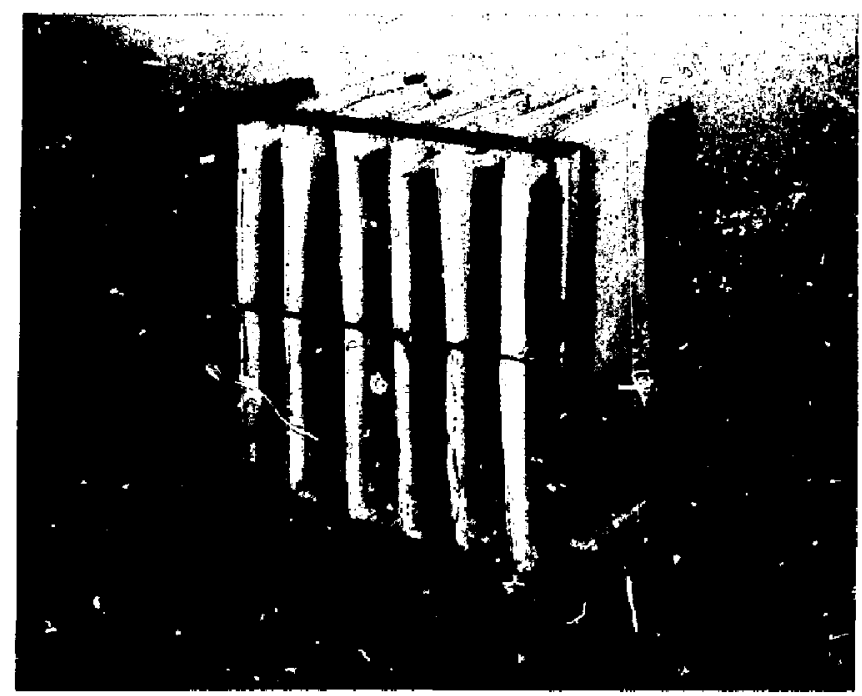

Figure 13. Disposable electrofibrous prefilter showing the inlet side.

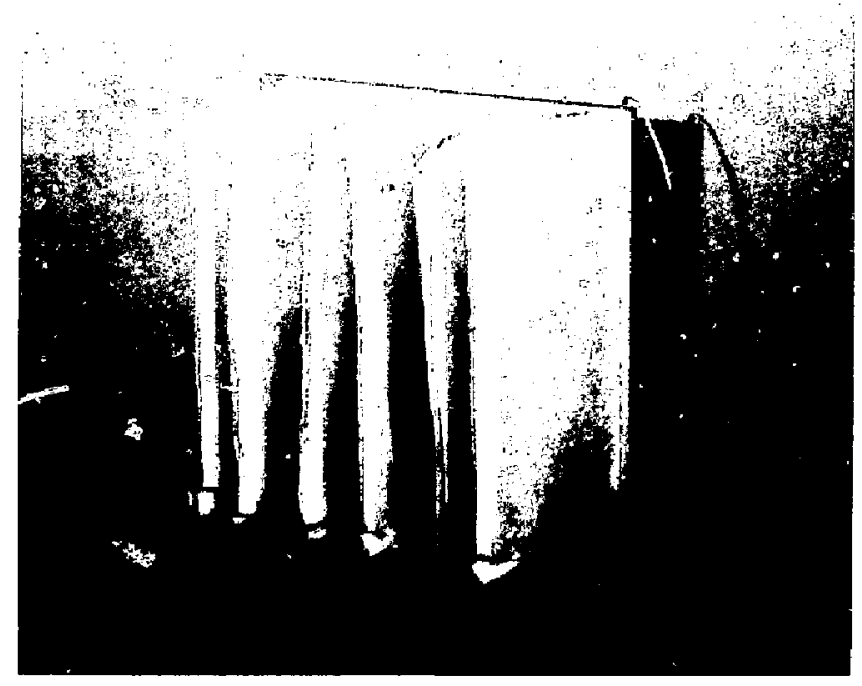

Figure 14. Disposable electrofibrous prefilter showing the exit side. 
Since the electrodes and sealing frame are permanently glued to the filter medium, when the filter medium becomes plugged the entire electrofibrous filter must be replaced, hence its designation as a disposable prefilter. Figures 15 and 16 show the disposable prefilter being mounted in the filter housing. The disposable prefilter was mounted on a transparent plastic frame in Figs. 15 and 16 to allow visual inspection of the installed prefilter. In field applications the sealing frame of the disposable filter would be mounted flush against a flange on the ventilation duct.

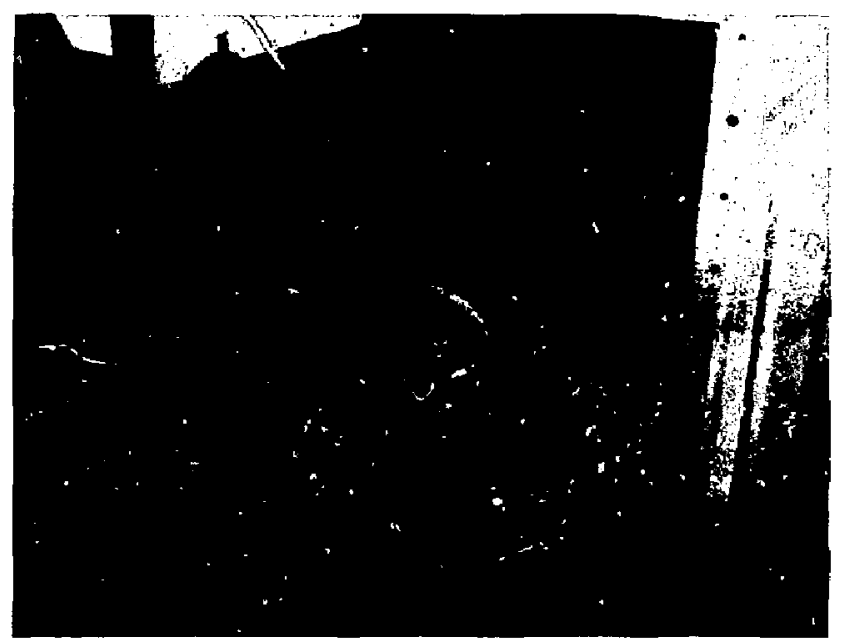

Figure 15. Disposable electrofibrous prefilter being mounted in the filter housing.

The replacement of the disposable filter is easier in some respects and more difficult in others when compared to replacing the filter medium in the permanent filter model. Mounting the disposable filter on the sealing flange of a ventilation duct is much easier than replacing the filter medium in tise permanent filter model. However, since the disposable filter has a rigid frame and integral electrodes, the disposable filter is more buTky and hence more difficult to pass through the entry-exit ports when compared to the filter medium for the permanent filter. 


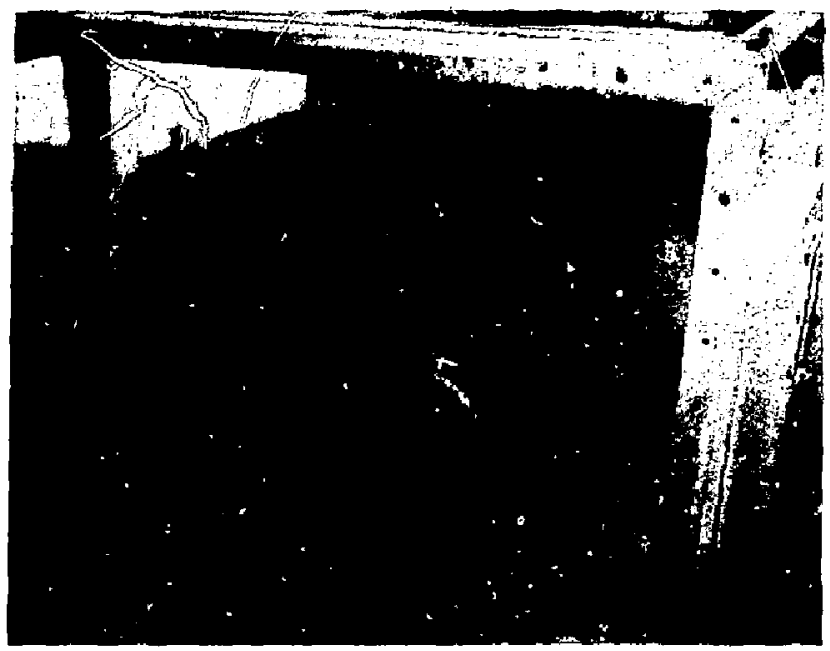

Figure 16. Disposable electrofibrous prefilter mounted in tire filter housing.

Both stationary electrofibrous prefilter designs use the same high voltage power supply shown in Fig. 17. He designed the high voltage power supply to convert 100 volt $A C$ input to $10 \mathrm{kV} D C$ output. Figure 18 stiows the top view of the power supply with the cover removed. The major component in this unit is a solid state power supply obtained from Spellman. It is shown as the rectariguTar box in the lower right hand corner. Since the power supply cannot exceed $70 \mu \mathrm{A}$ output current at $10 \mathrm{kV}$, the unit poses no hazard from electrical shock. One can touch the high voltage output and not feel the electrical discharge. The power supply shown in $\mathrm{Fig} .17$ has separate push button switches to energize the unit and to supply high voltage output. The high voltage switch also resets the high voltage output if the power supply was overloaded; i.e., if the load on the power supply exceeded $70 \mu \mathrm{A}$. Indicator lights on the front panel show if the high voltage is on or off.

We designed and built two different models of the stationary e lectrofibrous prefilter to accomodate the wide range of applications anticipated in the nuclear industry. The primary factor controlling the madel to be used is the frequency of filter changes. The permanent filter model would be used in applications requiring a frequent replacenent of HEPA filters, while the disposable filter model would be used in applications where HEPA filters are changed less frequently. Applications where the HEPA filter is rarely changed would, of course, not benefit from any prefilter. Figure 19 illustrates how the frequency of changing HEPA filters affects the total filtration cost of the permanent and disposable filter models. The disposable 


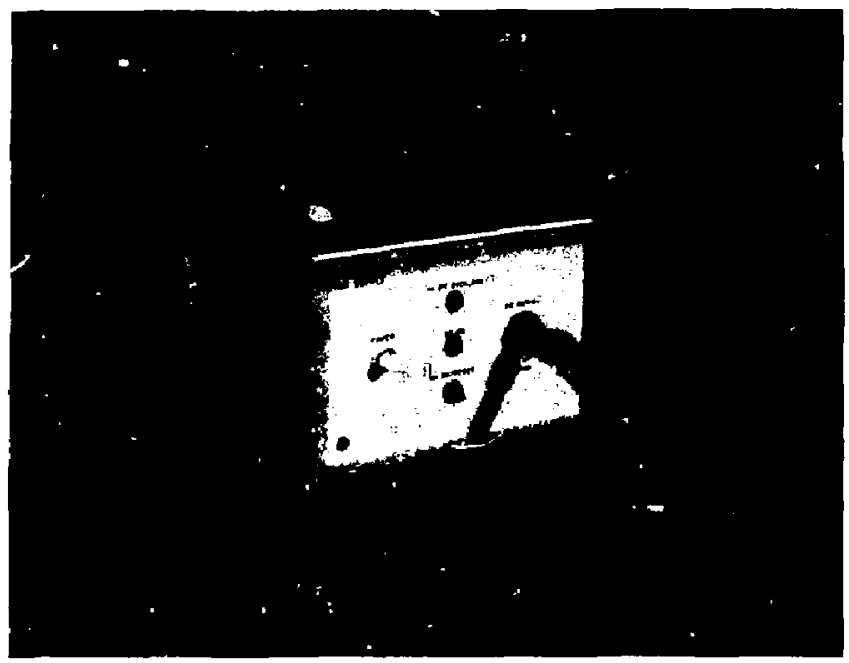

Figure 17. High voltage power supply.

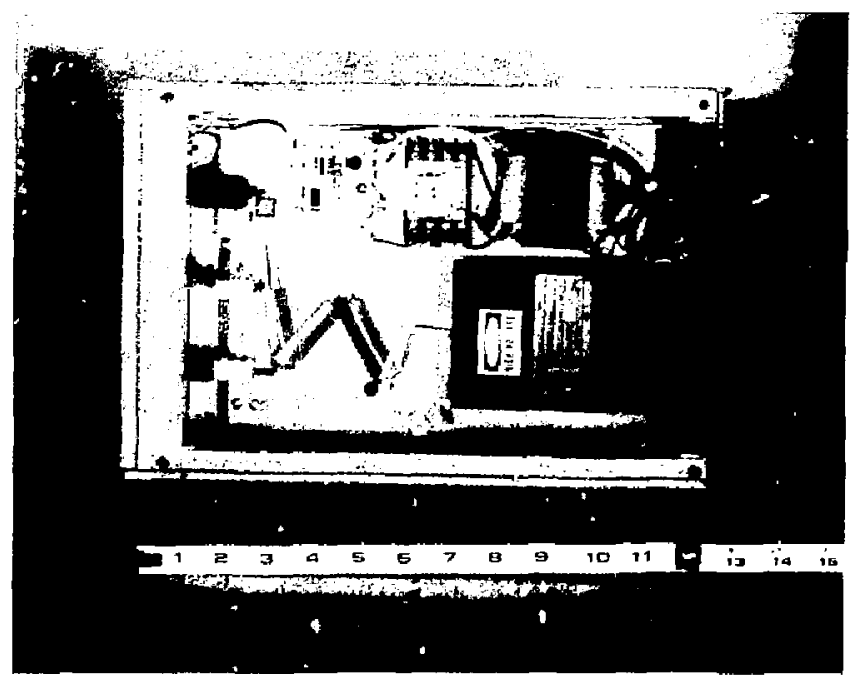

Figure 18. High voltage power supply with top cover removed. 
electrofibrous prefilter has a low fixed cost due to the high voltage power supply, but a $h$ igh operating cost since the entire filter is replaced. In contrast, the permanent electrofitrous prefilter has a high fixed cost due to high voltage power supply plus the permanent filter, but a low operating cost since only the inexpensive filter mediun is replaced. Figure 19 shows that the disposable filter model will result in lower filtration costs than the permanent filter model in applications where HEPA filters are changed less frequently. The opposite conclusion is reached in applications where HEPA filters are frequently changed.

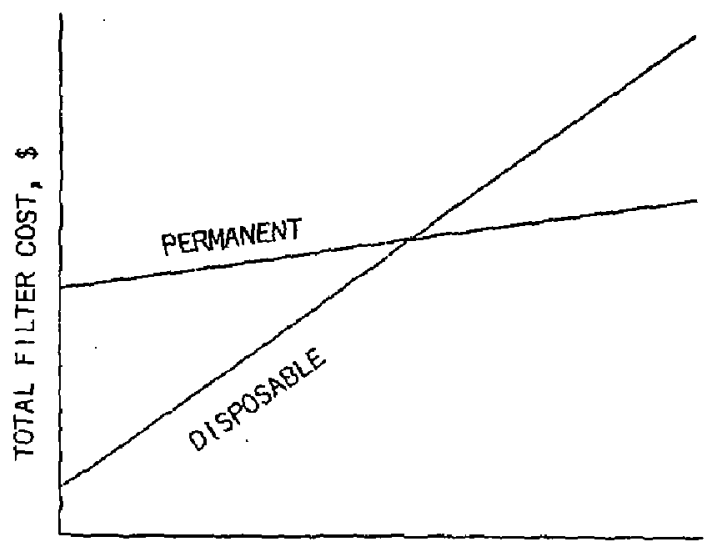

FILTER CHANGES / YEAR

Figure 19. Total filtration costs as a function of filter changes per year for the permanent and disposable filter models.

\section{Laboratory Evaluation of Stationary. Electrofibrous Prefilter}

We cenducted a laboratory evaluation of the disposable prefilter design in our large-scale filter test facility shown in Fig. 20 . This facility allows us to evaluate the performance of prototype filters under controlled laboratory conditions at flow rates up to $1300 \mathrm{cfm}$. Figure 21 shows the filter efficiency of the disposable prefilter design as a function of applied voltage at two air flow rates. The filter efficiencies were determined using sodium chToride aerosols generated with a Wright nebulizer and measured with a light scattering photometer. The $\mathrm{AaCl}$ aerosols have an aerodynamic mass medium diameter of $0.8 \mu \mathrm{m}$ with a $a \mathrm{~g}=2.0$. Figure 21 shows that the filter efficiency increases significantiy with increasing voltage at both flow rates, al though the lower flow rate shows the greater increase. 


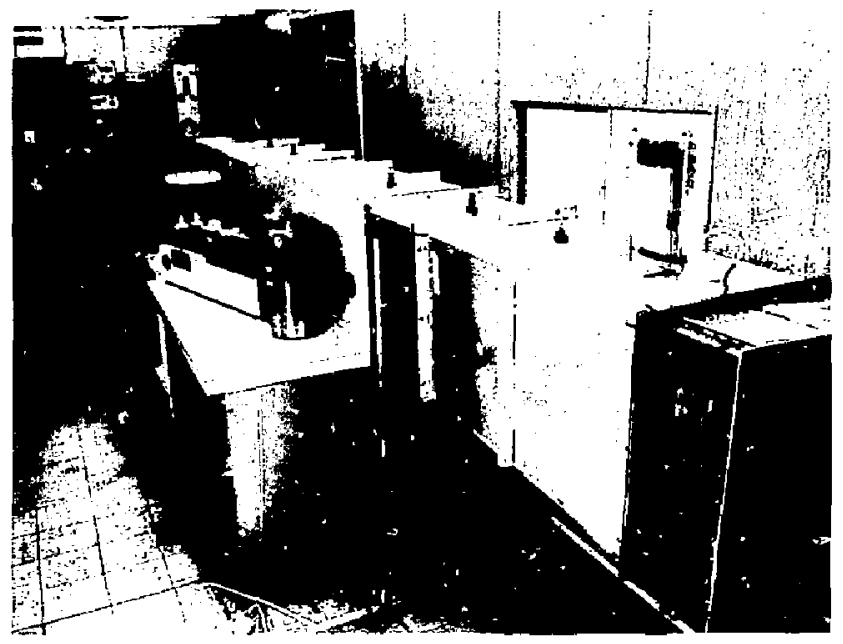

Figure 20. Large-scale filter test facility.

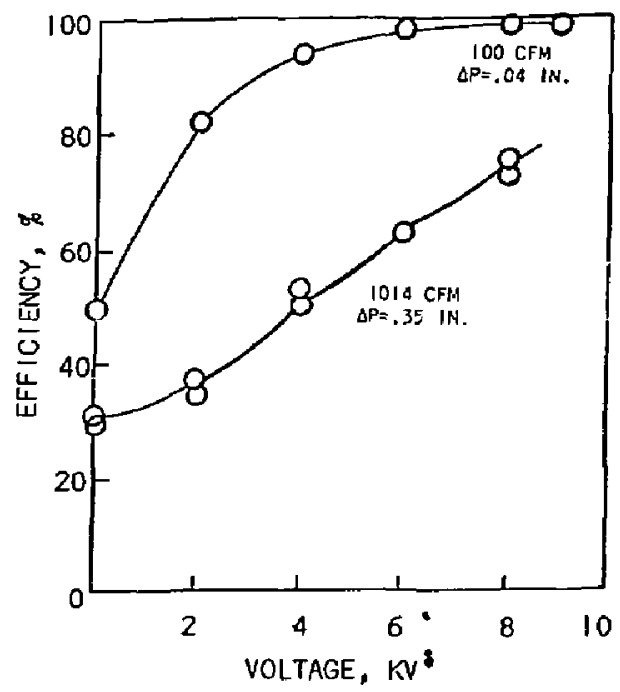

Figure 21. Efficiency of disposable filter as a function of applied voltage at 100 and $104 \mathrm{cfm}$. 
The effect of air flow on filter efficiency and pressure drop for the disposable filter is shown in Fig. 22. This figure shows that higher efficiencies and lower pressure drops are obtained at lower air flows. The two efficiency curves represent the filter efficiency with $0 \mathrm{kV}$ and $8 \mathrm{kV}$ applied to the filter electrodes. The efficiency of the electrofibrous prefilter is much higher at the lower afr flows because of the increased residence time that permits the electrical farces to attract particles.

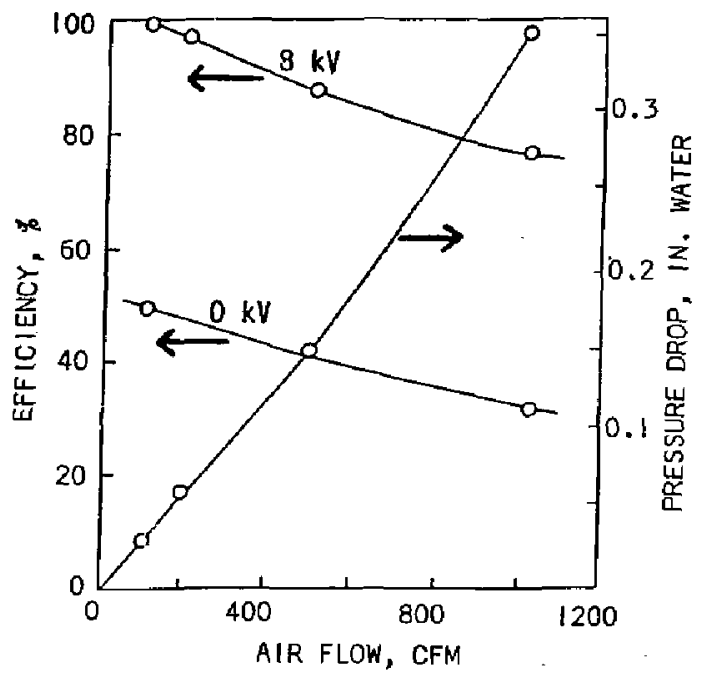

Figure 22. Filter efficiency and pressure drop as a function of air flow for the disposable electrofibrous prefilter.

D. Field Evaluation of Stationary Electrofibrous Prefiłters in LLNL's Uranium Burn Box

We then made preparations to install the electrofibrous prefilter in LLNL's uranium burn box. He selected this box for our field eva'luation because it had one of the $h$ ighest rates of radioactive particulate emissions at LLNL and would therefore provide fielo data within a relatively short $t$ ime. Figure 23 is a photograph of the uranium burn box before we modified it for our field evaluation. The uranium burn box was designed to oxidize machine turnings and chips of depleted uranium metal to convert it into a stable form for shipment and burial. If the uranium turnings were not stabilized they would ignite and burn when exposed to oxygen. Ur anium turnings are brought to the facility in barrels filled with water to prevent premature oxidation. 


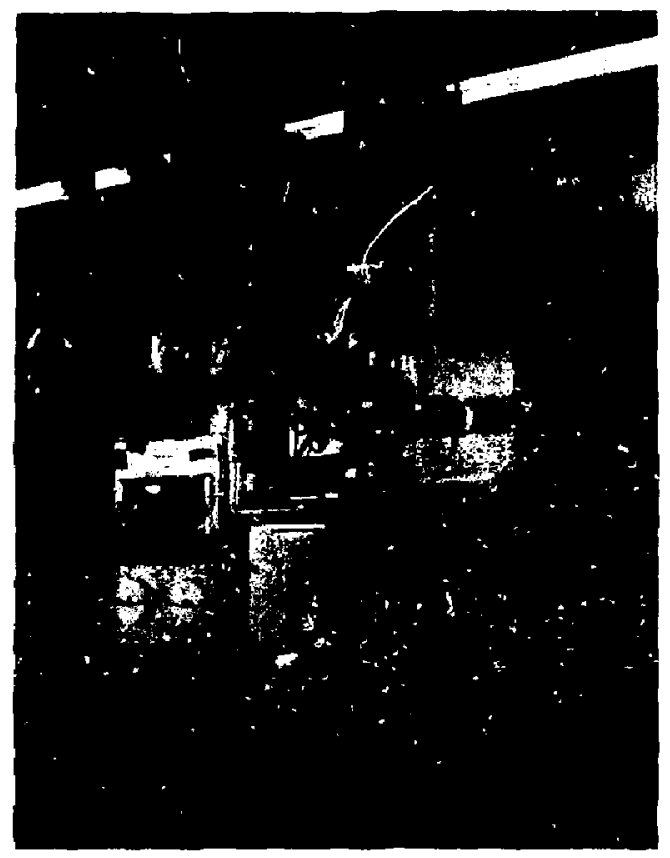

Figure 23. Uranium burn box at LLNL prior to our field evaluation.

The first step in the operation of the burn box is to laad a barrel into the left side of the burn box as shown in Fig. 24 . The barre 1 is then mounted on a pivot and the 1 id removed as shown in Fig. 25. After the access door is closed, the box is purged with an argon atmosphere. The contents of the barrel are then poured into a tray located beneath the barrel that sieves out the turnings from the water/oil mixture. Since the barrels often cantain sludge, the turning are usually washed with water. The tray of uranium turnings is then moved into the center chamber of the burn box (see Fig. 23) where the turnings are ignited with an electric arc and burned in a controlied fashion by adding oxygen. After the oxidation is complete and the tray cooled down, the tray is then transferred to the chamber on the right in Fig. 23. Here the ash is transferred to disposal barrels by air suction.

Each of the three chambers in Fig. 23 has a separate exhaust and HEPA filter that connect to a common exhaust 1 ine. The exhaust from the two end chambers passes through a $500 \mathrm{cfm}$ HEPA filter mounted on top of the respective chambers. A heat exchanger inside the center chamber cools the exhaust before 
passing through two $135 \mathrm{cfm}$ HEPA filters. The exhaust from this chamber is saturated with water vapor and has an extremely high particulate loading. These severe conditions would cause the two $135 \mathrm{cfm}$ HEPA filters to plug after about three days of operation and force the operation to be shut down until the filters were changed. An examination of the HEPA filters showed they had a heavy particle deposit and were saturated with water

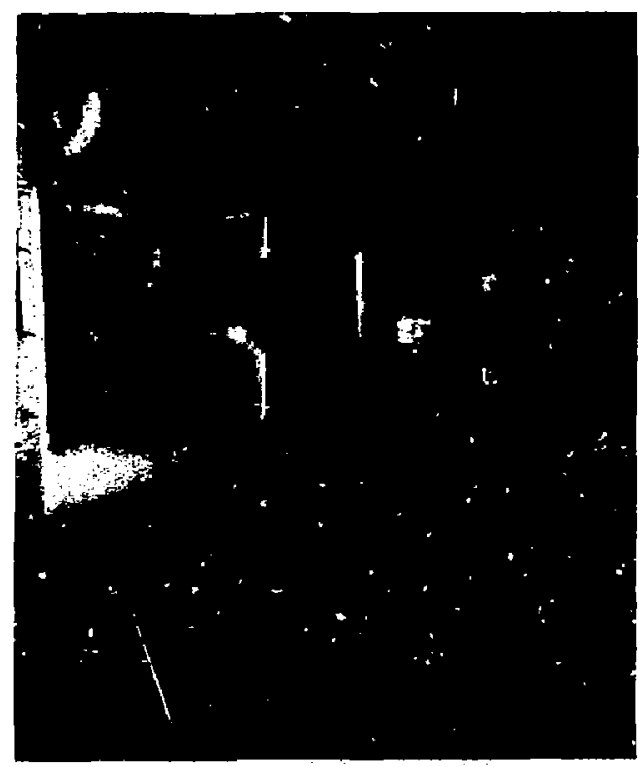

Figure 24. Operator loading a barrel of uranium turnings into the burn box.

Except for the saturated water in the exhaust, the uranium burn box appeared to be a good choice for evaluating our electrofibrous prefilter. However before we could install our r'-ctrofibrous prefilter we had to reduce the water vapor in the exhaust. He e sperimented with a number of different schemes for chilling the exhaust to remove the water vapor but were not successful. The problem was due to the high particulate concentration causing the water separator to plug. Cooling the exhaust would form a mist of very small water droplets that could not be removed from the gas strean without also removing the uranium oxide particles. The only effective means for removing the water drops was a demister pad or a coarse filter that would also remove the uranium oxide particles and subsequently plug the filter. Since dehumidifying the exhaust would merely transfer the plugging problem from the HEPA filters to the water demisters, we abandoned this approach. 


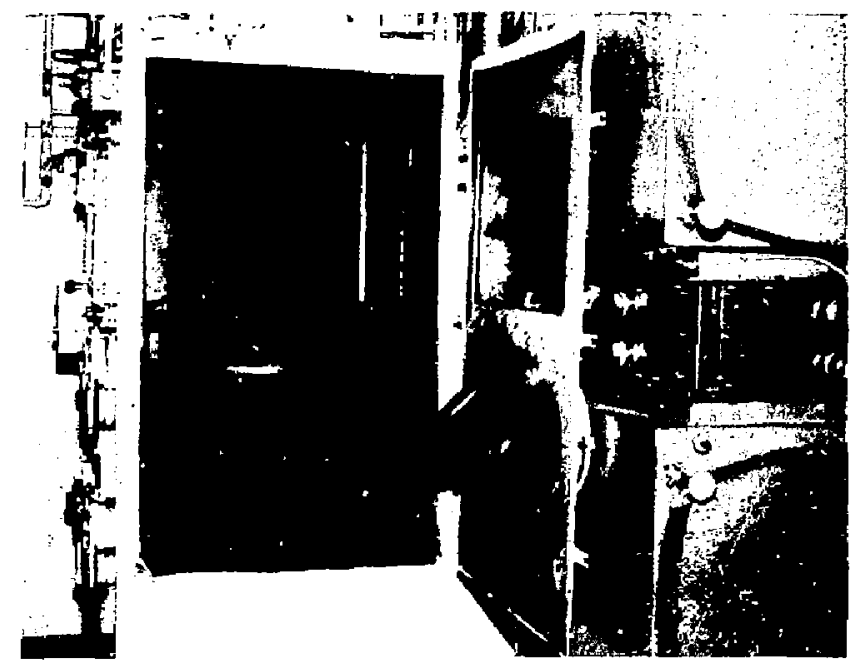

Figure 25. Barrel of uranium turnings mounted on a pivot inside the burn box.

At the suggestion of Dr. T. R. Thomas $(6)$ we built a dilution system for reducing the relative humidity of the exhaust by dilution with room air. With the added dilution air, we increased the exhaust flow from about $60 \mathrm{cfm}$ to $250 \mathrm{cfm}$. Further dilution was not possible without an extensive modification of the building ventilation system. To reach a reasonable level of relative humidity, we then added a heater to the dilution air. Adding hot dilution air to the exhaust from the burn box tiius provided a reasonable environment for evaluating our disposable electrofibrous prefilter.

We then built and installed a special ventilation system shown in Fig. 26 for evaluating our electrofibrous prefilter. ihe ventilation system consisted of two HEPA filter housings obtained from MSA and appropriate transition ducting. A sufficient number of viewing ports were added to the transition ducts to permit visual inspection of the prefilter and HEPA filter. We also; added pressure gauges to measure the pressure drop across the prefilter and. HEPA filter, a hot wire probe to measure the total exhaust flow and a light scattering photometer to measure the efficiency of the prefilter. This diagnostic equipment enabled us to evaluate the performance of the prefilter on a routine basis. We will also periodically measure the size distribution of the uranium oxide particles using additional instruments.

We then installed the disposable electrafibrous prefilter in the exhaust ventilation system as illustrated in the following sequence of photographs. Figure 27 shows two operators removing the cover off the top filter housing. The electrofibrous prefilter is then lifted into the filter housing in Fig. 28. Note that the disposable prefilter in mounted in a clear plastic frame. 


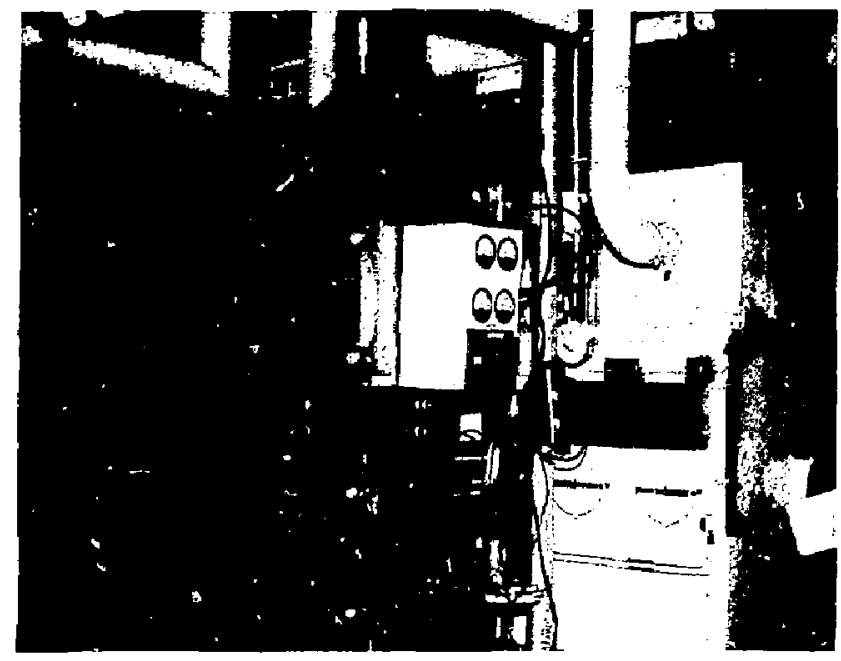

Figure 26. Ventilation system built for evaluating the disposable electrofibrous profilter.

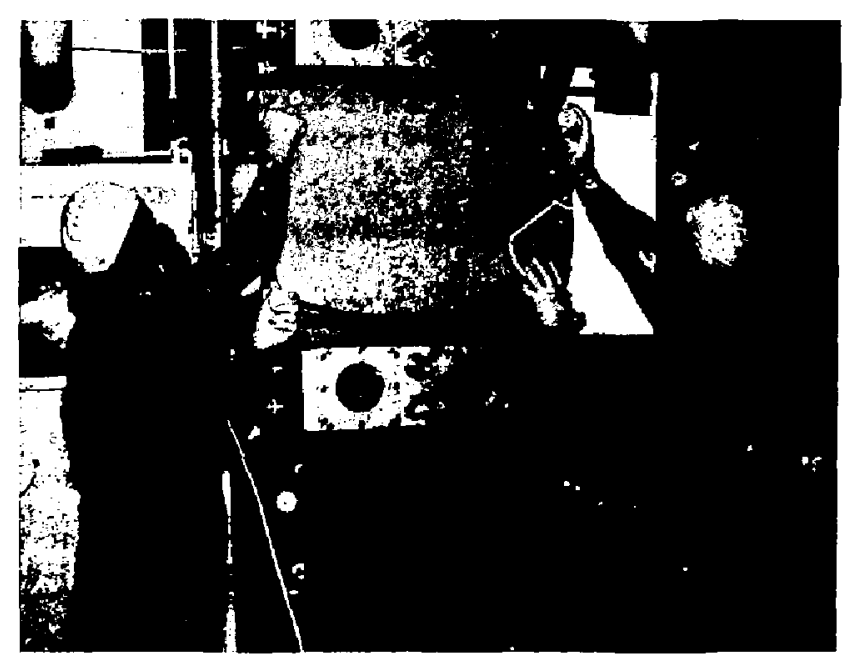

Figure 27. Operators removing the filter housing cover. 


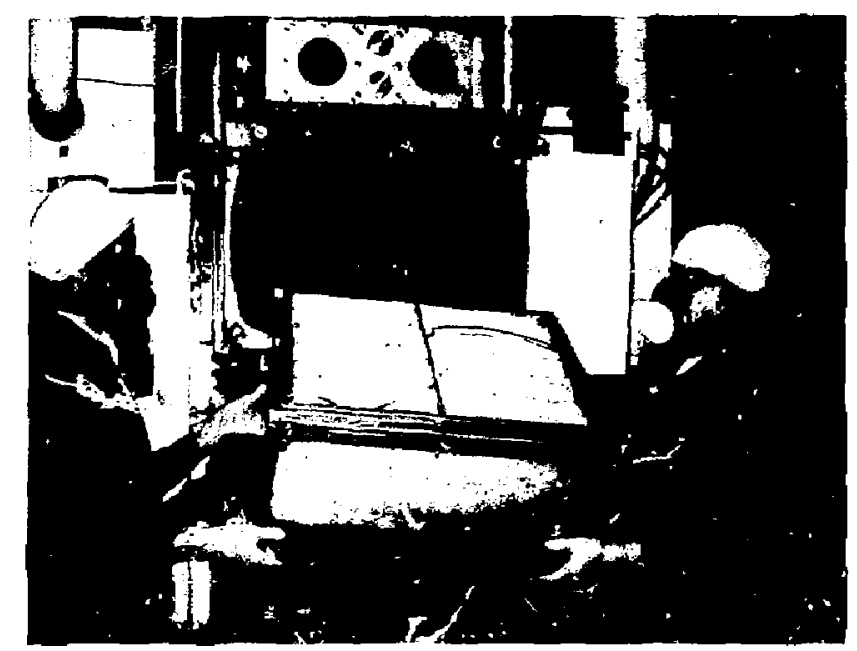

Figure 28. Disposable electrofibrous prefilter being inserted into the fiiter housing.

This was necessary since the filter hous ing was intended for HEPA filters. Before the prefilter was pushed into the filter holder, the high voltage lead from the filter was connected to the high voltage cable inside the housing (Fig. 29). The prefilter was then clamped against the sealing flange in the filter housing ( $F \mathrm{ig}, 30$ ) and the cover replaced over the filter housing. Figure 31 shows the electrofibrous prefilter installed inside the filter housing as seen through one of the viewing ports. Note the $h$ igh yoltage lead extending from the electrofibrous prefilter to a throughput on the ducting wall. The horizontal rod in the photograph is the upstream aerosol sample line that can traverse the width of the filter housing.

We then evaluated the performance of the disposable electrofibrous prefilter during a normal burn operation. The experimental results obtained from the first burn following the prefilter installation is shown in Fig. 32 . Th is figure shows the efficiency of the prefilter in trapping uranium oxide particles with and without high voltage. We measured the filter efficiency without high voltage by periodically turning off the high voltage power suppty. The high voltage increases the fitter efficiency from about $95 \%$ to 99\%. This represents an 80\% reduction in the particles reaching the downstream HEPA filter. During the 50-minute test the pressure drop across the prefilter increased from 0.17 inches to 0.35 inches of water. The large spike in current across the high voltage and ground electrodes was presumably caused by an excess ive amount of water vapor generated during the start up of the burn. 
We $p l a n$ to monitor the performance of the prefilter in a similar fashion to establish the particle loading capacity of the prefilter and extension in HEPA service life. Since the uranium axide particles appear to form a rather loose deposit on the filter surface, we will also explore methods for cleaning the prefilter for reuse. The extremely high particulate emissions from the burn box will certainly provide a considerable amount of data to exaline a number of questions regarding the use of stationary electrofibrous prefilters in nuciear ventilation systems.

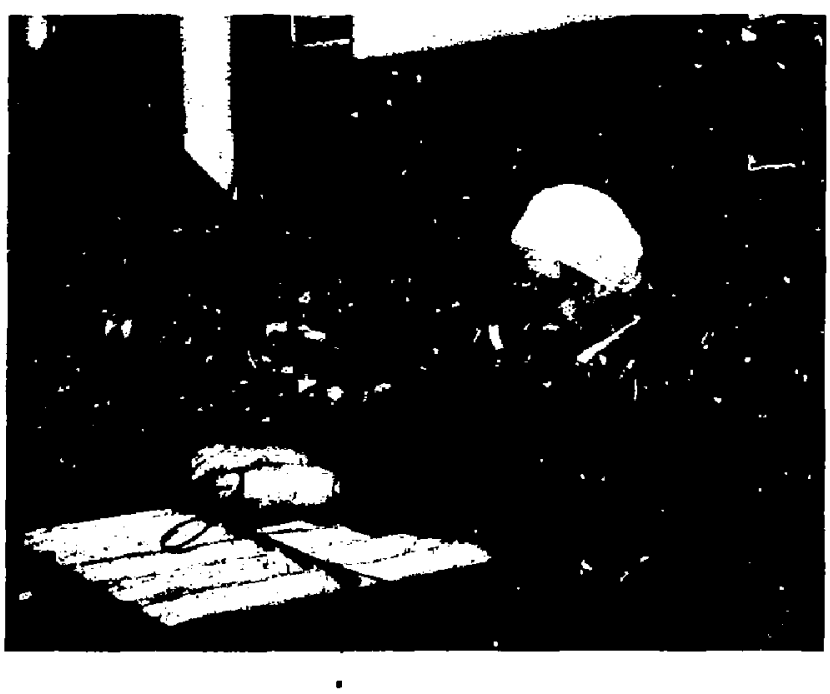

Figure 29. Operator connecting the ligh voltage lead from the filter to the high voltage cable inside the filter housing. 


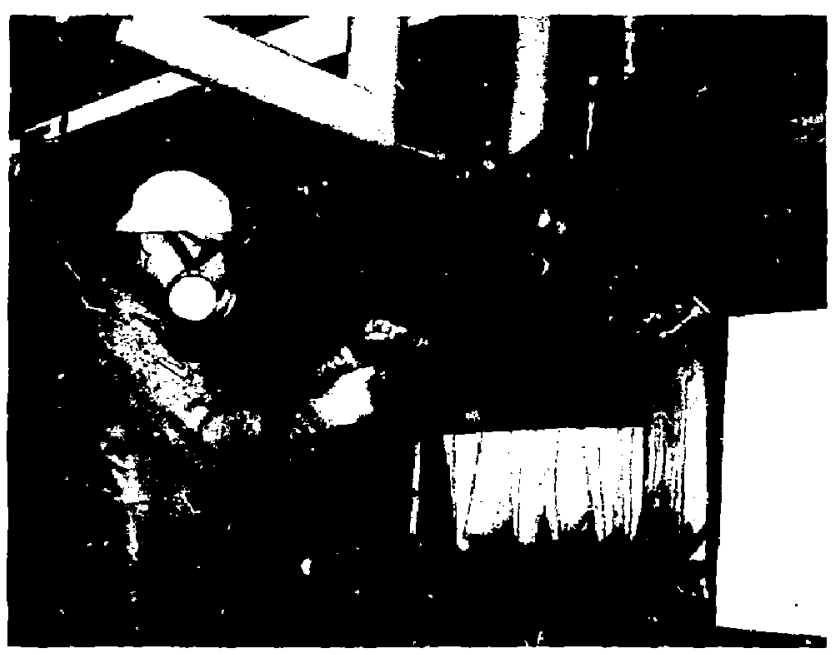

Figure 30. Operator clamping the prefilter against the sealing flange.

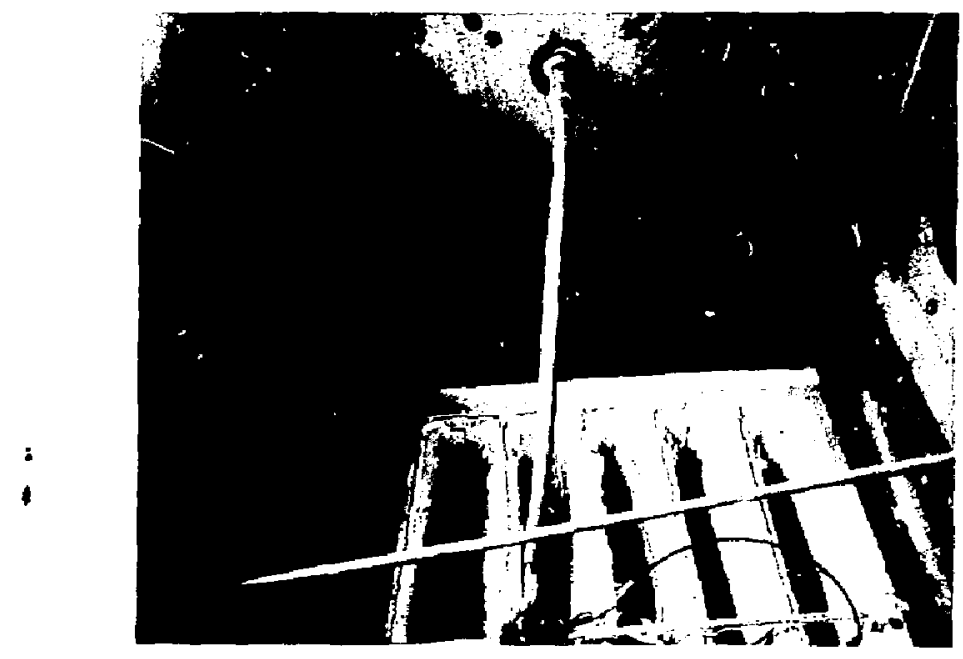

Figure 31 . Disposable electrofibrous prefilter installeo in the filter housing. 


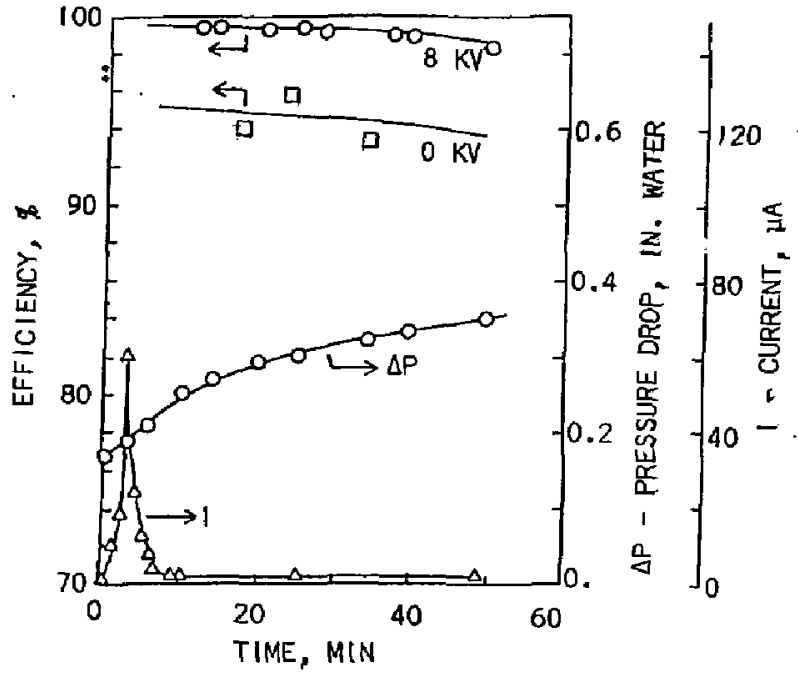

Figure 32. Performance of disposable electrofibrous prefilter during a uranium burn operation. 


\section{II1. Evaluation of Rolling Electrofibrous Prefilter at LLNL's Fire Test Facility}

A. Introduction

One of the most serious accidents that can occur in a nuclear facility is a fire. A critical factor that increases the severity of a fire is the potential loss of the ventilation system due to the plugging of HEPA filters by smoke aerosols. Alvares et al ( 7 ) have summarized the results of a ten-year study to develop countermeasures to protect HEPA filters from heat and smoke generated by fire. They identified the following three countermeasures to mitigate the problem:

1) materials management to reduce the quantity of combustible materials,

2) conventional fire decection and fire suppression techniques,

3) smoke removal or treatment in the ventilation duct upstream of the HEPA filter.

Among various methods examined for in-duct co itermeasures were wet scrubbers, afterburners, granular beds and prefilters. Although efficient prefilters appeared to stop the smoke aerosols, they were also rapidly plugged. Because of the tar-like property of the smake aerosols, cleanable prefilters, like those used in bag houses, would not offer any benefit. Once the smoke aerosols deposited on a filter, the particle deposit could not be removed.

The concept of a rolling electrofibrous prefilter was developed for use in ventilaton systems in the nuclear industry to prevent the plugging of HEPA filters during a fire episode, while also extenoing the lifet ime of HEPA filters during normal use. During normal conditions, the rolling electrofibrous prefilter would behave in a manner similar to the stationary electrofibrous filters described in the previous section. We have electrified the rolling prefilter since it results in a higher collection efficiency and longer filter life than a conventional fibrous filter because of the added e lectricel capture mechanisms. $(1,3,4)$ The rolling prefilter (RPF) concept allows for the continuous replacement of filter media ance it has plugged. The media can be advanced automatically subject to the pressure loss across the filter. However, since the probability of having a $f$ ire is very low, the multi-pass RPF would function primarily as a stationary prefilter. From an operational point of $v i e w$, the RPF would significantly reduce maintenance costs associated with replacing stationary prefilters, but would require more space, and cost more than our stationary electrofibrous prefilter. The high initial cost of the rolling electrofibrous prefilter would be offset by the significantily reduced maintenance costs for replacing stationary prefilters.

The application of the electrofibrous RPF in a nuclear ventilation system would have to be determined on a bas is of a cost-benef it study and a risk analyses of the fire hazard. For applications where the maintenance cost is very high, the RPF would be justified in a cost benefit study even if the risk is very li $\mathrm{W}$. In this case, the savings in maintenance costs will off set the capital cost of the RPF. The RPF would also be justified in ventilation systems that have high fire $r$ isks even though the maintenance casts are very low. However, if a given ventilation system has a low maintenance cost and a low fire risk, then the use of a RPF would not be justified. 
The simplest version of an RPF is a single-pass unit. In previous studies $(5,9)$, we showed that a single-pass RPF was effective in protecting a HEPA filter from plugging by a concentrated smoke aerosol for up to 60 minutes, or the complete duration of the fire. Unfortunately, the single-pass design cannot be used for a stationary electrofibrous prefilter that operates under normal conditions because of excess ive pressure drop and face velocities that are encountered. Therefore, we have developed a pleated, or multi-pass, e lectrofibrous rolling prefilter.

\section{Design of the Rolling Electrofibrous Prefilter}

The design of an effective multi-pass, or pleated, rolling prefilter to protect a HEPA filter during a fire condition proved to be a much more difficult task than our experiences with the single-pass prefilter. The design of the multi-pass prefilter is far more complicated because of the pleats which the filter media must traverse as it moves across the ventilation ducting. The key feature of this design is a cantinuous screen belt that both provides support for the filter media and serves as the high voltage electrode. The filter media rides on the electrode belt and advances through the filter housing as the belt advances.

Since our first design of a multi-pass unit (5) we have made many modifications to reach the prototype described here. Changes have been made to address problems involving media tearing, media sticking, jamming of the transport chain, and edge leakage. Of these problems the edge leakage was the most critical and the most difficult to solve. We have reported our previous attempts at preventing edge leakage $(5)$ in which we explored a number of solutions involving deflection plates, compression seals and media modifications. We recently developed an edge seal design that produced sat isfactory results.

The design of the rolling electrofibrous prefilter described in this report, has incorporated a number of improvements over our previous design to upgrade the prefilter for use in a nuclear facility. An overall view from the front side of this prototype is shown in Fig. 33. In operation, the prefilter inlet duct (the central opening in $\mathrm{Fig}$. 33) would be connected to the upstream ventilation ducting. Through this opening, the filter media can be viewed traversing the ventilation duct a long three $V$-pleats. The left and $r i g h t$ ports in Fig. 33 expose portions of the take-up mechanism and storage box for the filter medium respectively. The rear view of the rolling prefilter is shown in Fig. 34. The central opening is the exit of the pleated filter section while the port on the lower right side allows access to the drive mechanism for the rolling prefilter. Plexiglas panels can be removed for access to these areas.

The control box located on top of the prefilter housing allows the selection of an appropriate pressure drop across the prefilter at which the filter media would be automatically advanced by the drive mechanism. In this manner, the filter medium which becomes loaded with particulate material can continually be replaced with clean medium. The filter medium would traverse from the filter medium storage box (right side of Fig. 33) through the pleated prefilter section (center portion) and be pulled out of the prefilter section by take-up rollers (left side of Fig. 33) and finally wound on a disposal spool. 


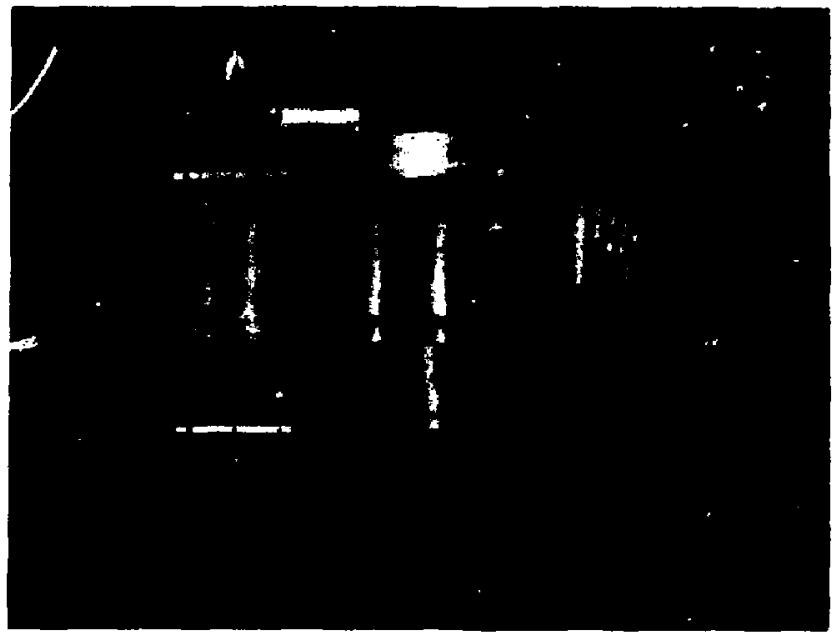

Figu e 33. Front view of rolling electrofibrous prefilter.

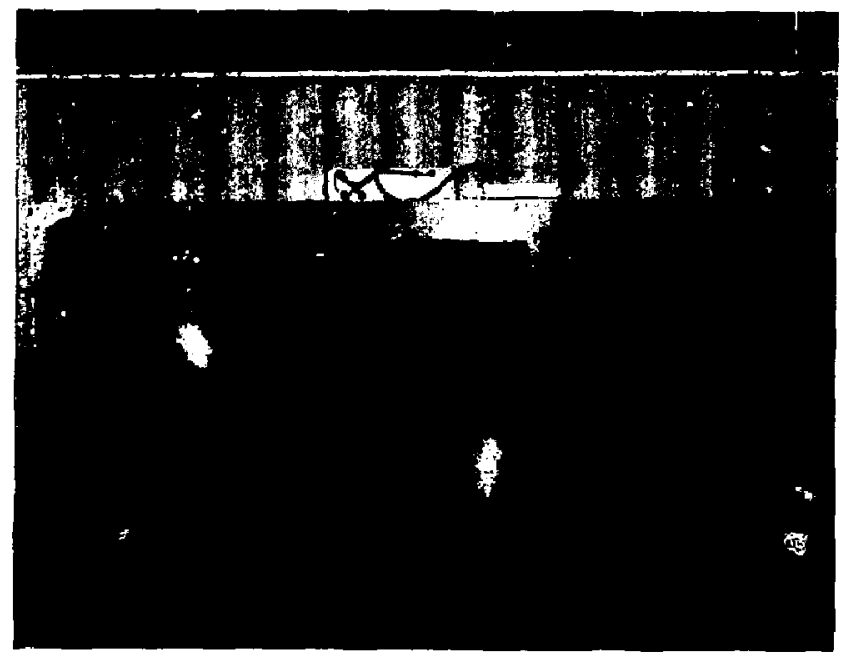

Figure 34. Rear view of rolling electrofibrous prefilter. 
Figure 35 shows an angie view of the rolling prefiter and shows the end of the filter storage box which has a capacity of 80 feet of filter mediun. Additional lengths of filter medium can be attached to the previous length. Access is provjded through the end port. A view from another angle of the roling prefifter in Fig. 36 shows the disposal spool for receiving spent media after it has lassed through the central filtering section. When the disposal spool has reached its capacity, the filter medium is cut and the spool removed from the disposal box and placed directly into a standard size waste drum. The cut filter medium is then attached to a new spool. A very important feature of our filter design is that fresh filter medium can be added and used filter medium disposed of while the roling prefilter is in operation.

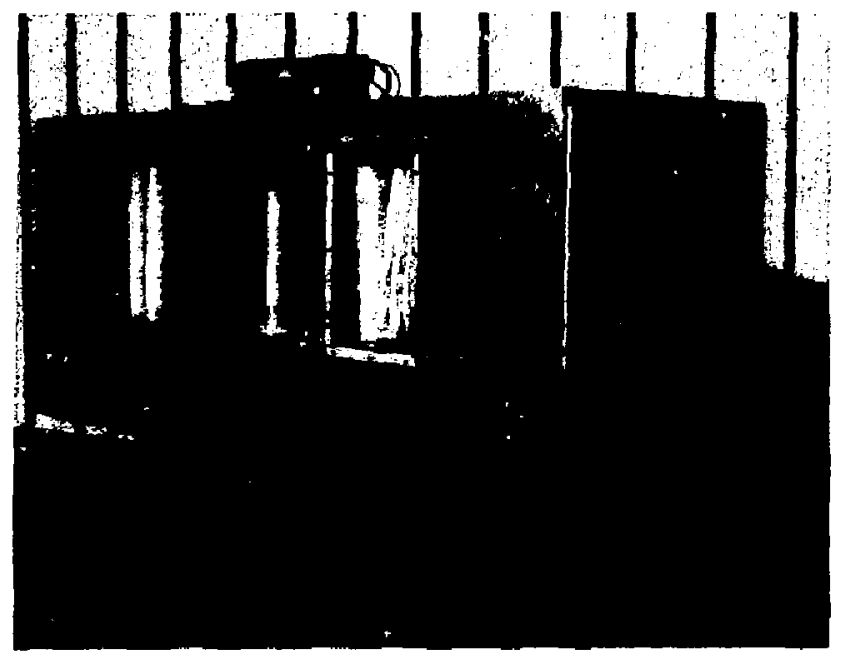

Figure 35. Rolling electrofibrous prefilter showing end view of filter media storage box.

The continuous belt which is used to support the filter medium can be seen in Fig. 34 through the exit duct on the downstream side of the prototype unit. The stainless steel belt consists of a wire mesh knitted over supporting rods that connect to a chain at both edges of the belt. Motor driven sprockets are connected to each chain and drive the belt around its path. Additional sprockets are used to provide pivotal points for changes in the belt direction. Ail of the sprockets are supported by shafts through the fjlter housing. The shafts penetrate the RPF housing and are supported on the outs ide panel by bearings. Previous tests showed that at increasing pressure drop, the screen belt becomes slightly deflected, and occasionally, one of the supporting rods would pull out from the chain links. We have also designed an optiona? stationary frame that supports the belt, thereby preventing distortion. We have not used this option in our rolling prefilter. 


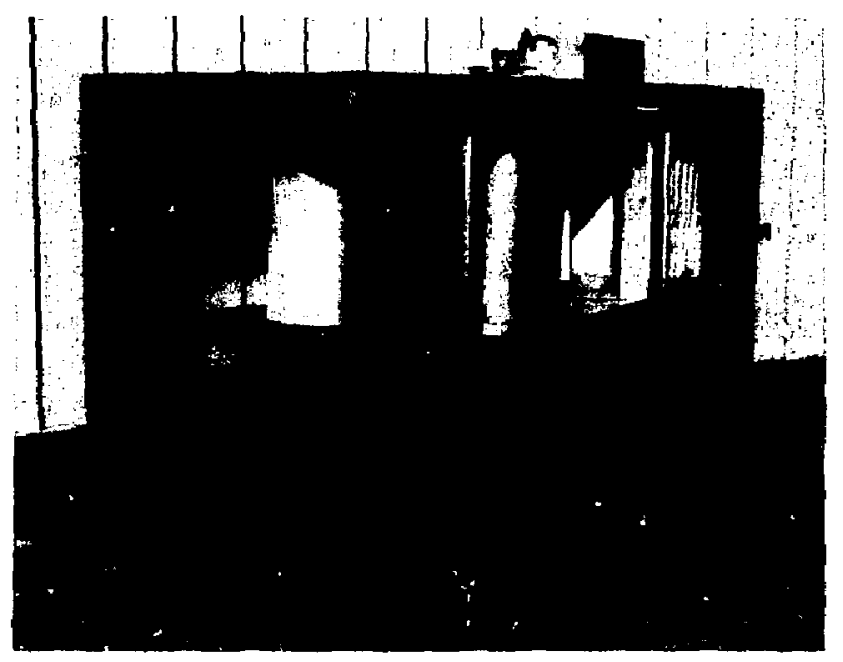

Figure 36. Rolling electrofibrous prefilter showing end view of filter media disposai spool.

The design of the electrofibrous RPF is further complicated by applying $h$ igh voltage to the screen belting. Special precautions are required to insure adequate insulation for the high voltage belt. The screen belting is insulated from the RPF housing by sheets of polyethylene or polycarbonate with specially cut grooves for supporting the belt chain. In addition to the screen insulation, all connections to the screen belting, like the sprocket shafts, are made from nonconduct ive material.

Figure 37 shows a front view of the rolling prefilter with the enclosure box removed. In this upgraded unit, three take-up rollers shown on the left side of the filter have been added to pull the media out of the pleated filter section and to improve guidance onto the filter medium disposal spool. The orive mechan isms for the take-up rollers and disposal spool are located below the disposal spool. Figure 37 shows the folded filter medium supported in a temporary fashion by a block of wood. The enclosure box has flanges tht normally provide support to the filter medium supply.

In previous designs of the rolling prefilter, leak paths around the filter edges were a serious problen. Edge leakage was especially bad around the curved portions of the filter path where the media is more compressed because of increased tension. To address this problem, several unsuccessful configurations were developed including narrow deflection plates and rope and rubber strip compression seals. In general, compression seals tended to overstress the filter medium and resulted in tearing. Our final design incorporates wide deflection baffles axtending out three inches upstream and four inches downstream of the filter media. Clase up photographs showing the central filtering section and the upstrean deflection baffles are shown in Figs. 38 and 39 respectively. The spacing between the upstrean and downstream baffles was determined by having the filter medium slightly compressed without significantly increasing the frictional drag. 


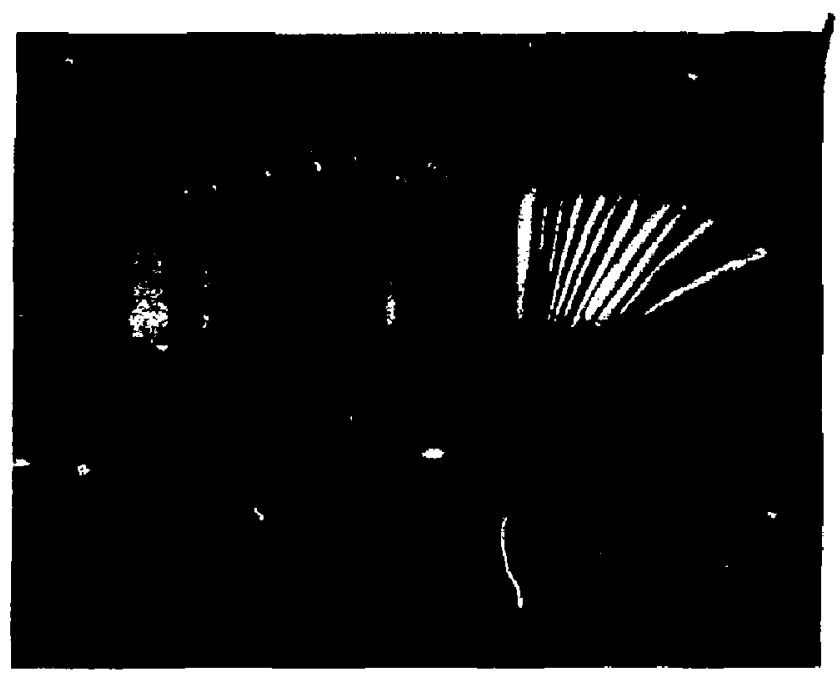

Figure 37. Front view of rolling electrofibrous prefilter with enclosure box removed.

Another important feature of the rolling prefilter design is the rollers that drive the filter medium around the rear pleats of the central filtering section. Figure 38 shows three rollers in the central filtering section. Our previous attempts at using smooth rollers proved to be unsuccessful because the sticky tar from the smoke aerosols would cause the filter medium to stick to the shafts and form a large ball which eventually stopped the drive mechanism. With short blades running along the roller length, the resulting rollers could grab the filter medium and drive it through the sharp turns without providing a sticking surface for the fitter medium.

The drive mechanisms for moving the electrode belt and filter medium have been thoroughly revamped in our upgraded unit as shown by the photographs in Figs. 40-42. Figure 40 shows the gear box and mator for the electode beit drive. This gear box also drives three take-up rollers that pull the filter medium out of the central filtering section as seen in Fig. 41 . The filter medium is pulled through exit slots connecting to the pleated filtering section by three take-up rollers that firmly grab the filter medium. Note the central roller has a number of blades that contact the filter medium. This is necessary to prevent the spent filter medium from sticking to the roller. In $F$ ig. 42 an additional motor ( $r$ ight and center) has been incorporated to maintain a constant torque on the filter medium as it winds on the take-up spool. Note the spring loaded bar below the disposal spool. It permits a rapid engagement or disengagement of the drive shaft when the disposal spool is replaced. A slot in the bottom end plate of the take-up spool allows the spool shaft to be removed. 


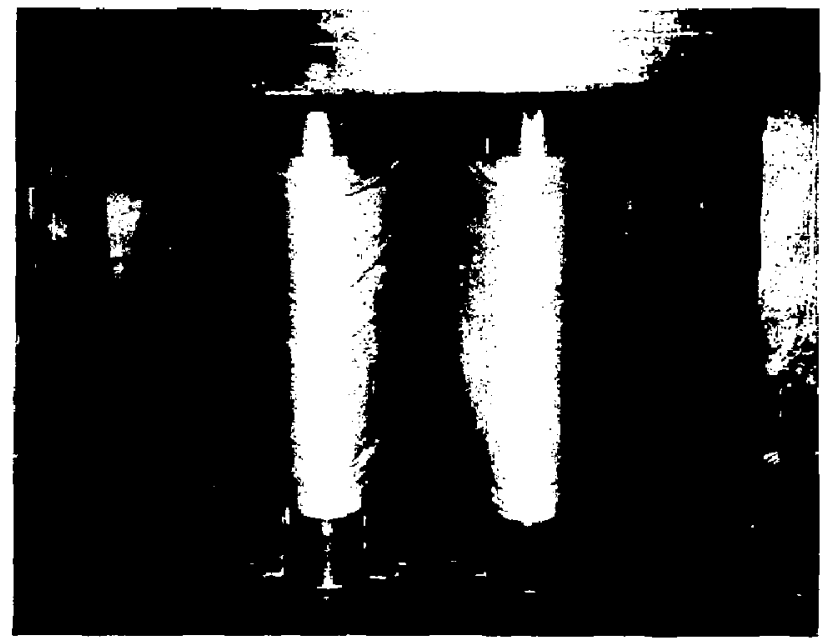

Figure 38. Central filterina section of rolling electrofibrous prefilter.

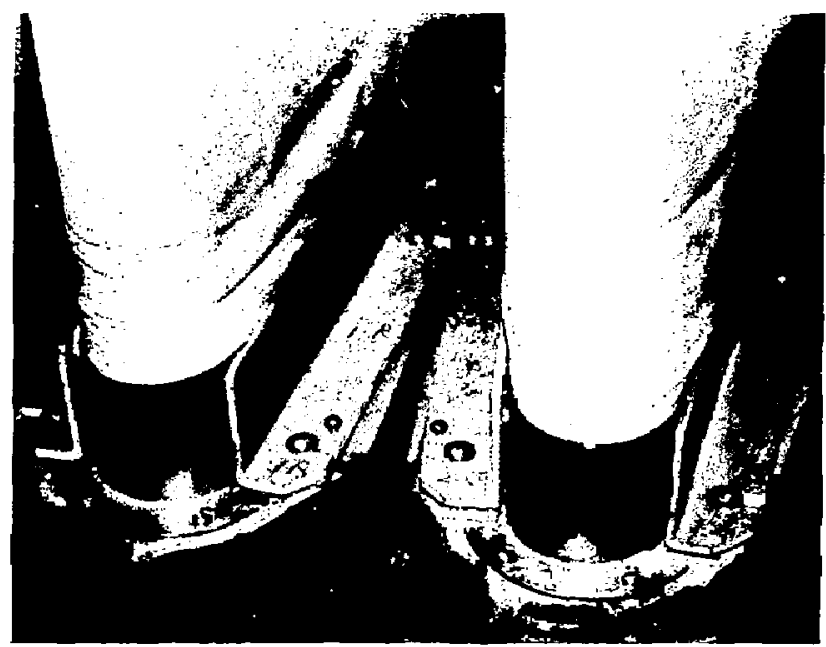

Figure 39. Upstream baffle of the filter edge seal. 


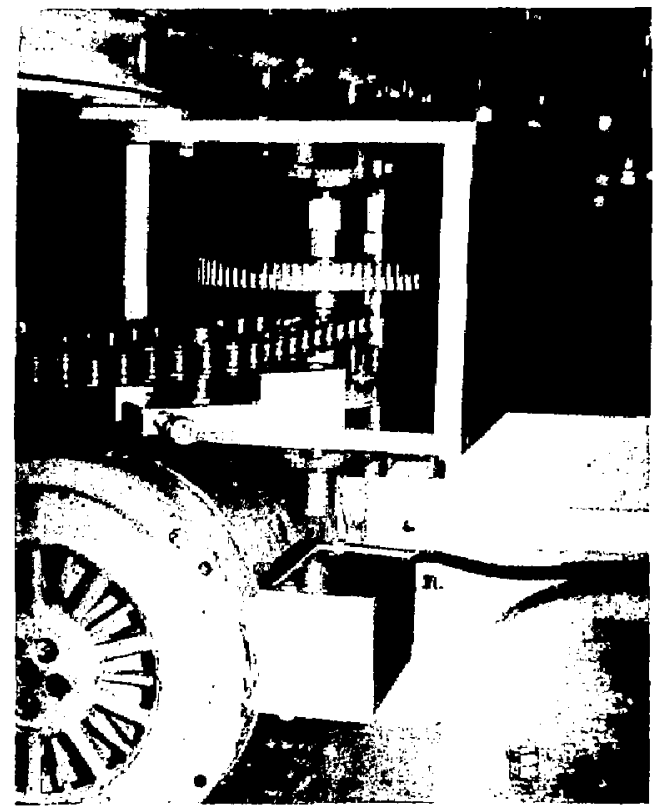

Figure 40. Photograph of electric motor and gear box that drives the electrode beit.

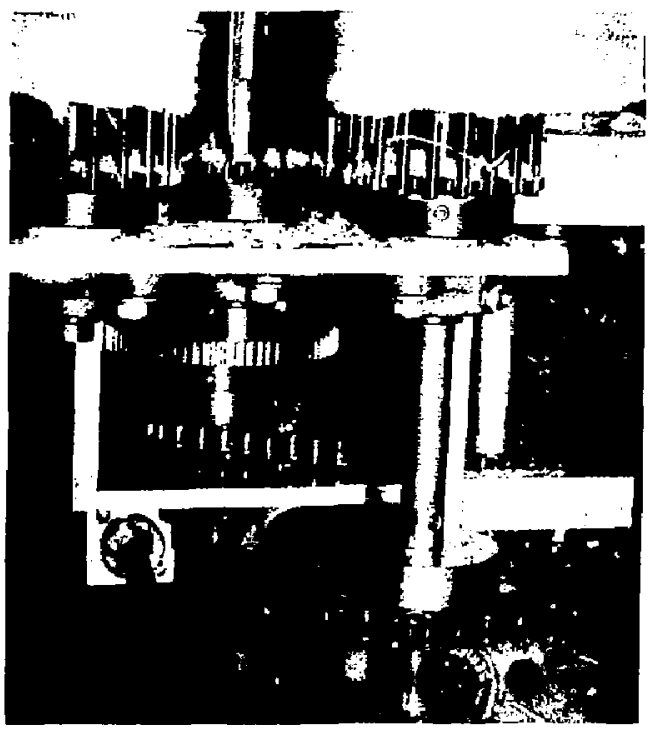

Figure 41. Photograph of the drive mechanism for the three take-up rollers that pull the filter media out of the central filtering section. 


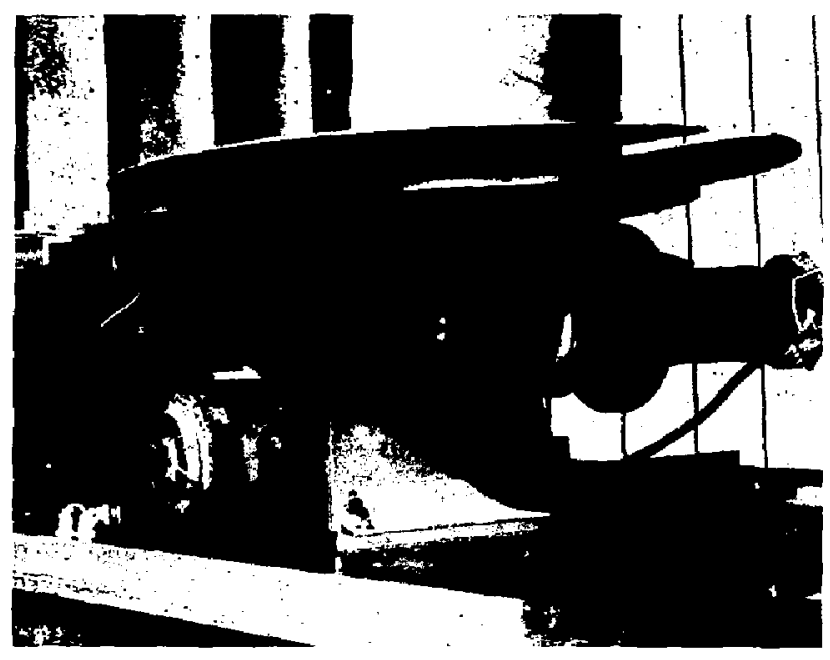

Figure 42. Photograph of the constant torque electric motor for driving the disposal spool.

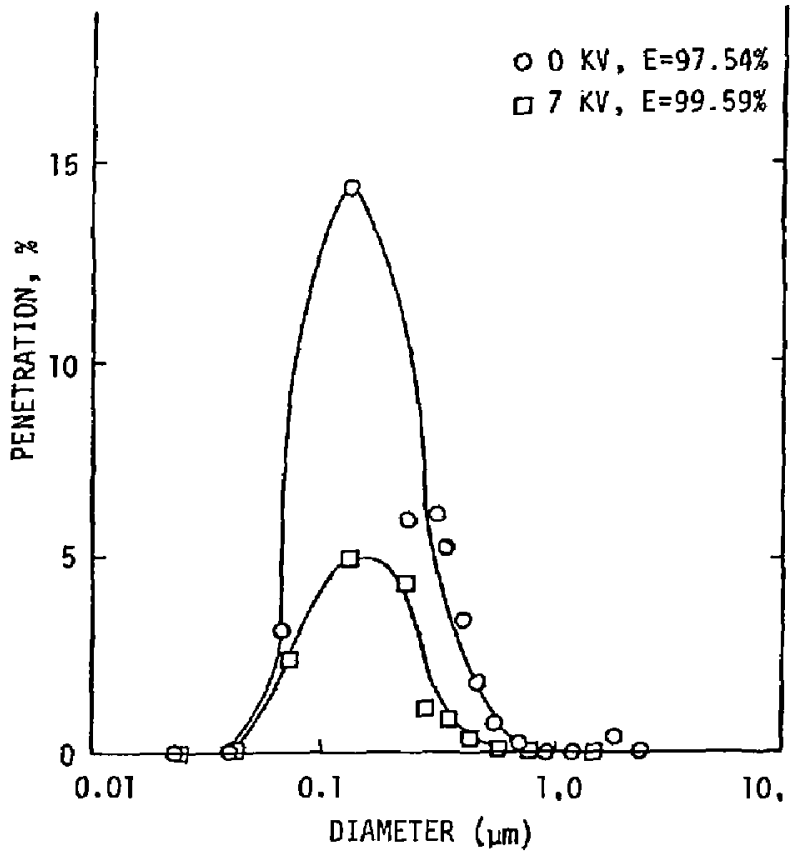

Figure 43. Penetration of DOS aerosols through composite media with and without high voltage as a function of particle size. 
The proper filter medium seiection proved to be a crucial aspect in achieving a successful fire test result. We could not continue to use the thick-laminated filter medium used in our single-pass prototype because of excessive spacing between the high voltage electrodes. Thinner filter medium of equal efficiency tended to lack the necessary strength for passage through the pleated filter. However, the major problem with a thin but efficient filter medium is the rapid saturation with liquid smoke particles which causes the filter medium to stick to the electrode belt and also decreases the filter efficiency. Also, we found that adhesives used to glue the scrim material onto the filter medium would melt and bind the filter medium to the moving screen. If the filter medium was too thick and porous, sufficient aerosol would pass through to the screen. The sticky properties of the callected particulates would again result in binding of the filter medium to the screen.

The filter medium used in the fire test described here is composed of five layers of Johns Mansville filter medium. These include, in order from upstream to downstream, two layers of AF-21, two layers of AF-4, and one layer of $A F-21$. The two layers of $A F-21$ were used on the upstream to provide a high loading capacity for liquid smoke aerosols and good resistance to compression. The two central layers of AF-4 were used to make the composite filter filter medium more efficient. A final layer of AF-18 was added to the composite filter to protect the AF-4 filter medium from being torn due to mechanical forces or due to sticking to tarry surfaces. To provide mare strength to this laminated structure, a scrim material is sewn to the filter medium on both sides. When the prefilter is used in an electrified mode of operation, an additional metal mesh screen (not shown) will be glued on the upstream side of the filter medium in place of the scrim material to serve as the ground electrode.

\section{Laboratory Evalution of Rolling Electrof ibrous Prefilter}

We conducted a laboratory evaluation of the filter medium using DOS aerosols at a face velocity of $22.5 \mathrm{~cm} / \mathrm{s}$. This face velocity corresponds to $500 \mathrm{cfm}$ through the rolling prefilter unit. Liquid DOS aerosols were used in this evaluation since smoke aerosols are predominantly liquid aerosols. Figure 34 shows the percent penetration of DOS aerosols through the composite filter medium as a function of particle diameter. This figure shows the percent penetration with and without an applied electric field. At a face velocity of $22.5 \mathrm{~cm} / \mathrm{s}$ the filter had a pressure drop of 2 in. water. Using a light scattering photometer we determined that the filter efficiency was $97.5 \%$ without an applied voltage and $99.6 \%$ with $7 \mathrm{kV}$ applied to the electrodes Electrifying the filter medium had therefore reduced the aerosol penetration by 837 .

We also evaluated the performance of the rolling prefilter by passing fresh filter medium through the filter housing several times and found that the operations of adding fresh $f i l t e r$ media and disposing of used filter media went very smoothly. The filter medium also tracked through our improved edge seal in the central filtering chamber quite smoothly. 


\section{Field Evaluation of Rolling Prefilter in Fire Test Cell}

Figure 44 is a drawing of the facility used tu evaluate the performance of our roliting prefilter in an actual fire episode. The facility is designed for fire exposure tests on containment ventilation systems and components. The facility consists of a negative-pressure ventilation test area (not shown in Fig. 44), the fire test cell, and the computer diagnostic room. The ventilation test area is coupled to the fire test cell by standard $2 \mathrm{ft} \times 2 \mathrm{ft}$ ventilation ducting. Diagnostic instrumentation at these locations is "hard wired" to a POP- 11 computer for data addressing, data reduction, and final display in hard-copy formut. Qetails and descriptions of the instrumentation and diagnostic equipment are contained in Ref. 7 and 8.

Figure 45 is a photograph of a composite crib used as the major fuel source for our tests. The fuel array is made of specific proportions of fue 1 materials common to physical science laboratories. We ignite the fuel with a natural gas burner that acts both as the ignition source and a thermal driver to maintain high constant temperatures in the crib when the test cell became ventilation controlied. This burn procedure results in reproducible fires and smoke aerosols for our filter evaluations. The smoke from the fire test cell is exhausted through a ventilation duct and is filtered first by the RPF and then oy the HEPA filter.

We installed the rolling electrofibrous prefilter in the exhaust duct of the fire test facilty. However, we did not operate the rolling prefilter in its electrical mode. Since the exhaust from the fire test cell is saturated with water and acid vapors they would rapidly short circuit our electrodes. At the time we conducted our fire test evaluations we had not completed all of the modifications to the rolling prefilter to upgrade it to nuclear standards. The fire test evaluation reported here was primarily intended to demonstrate that the pleated filtering section shown in $\mathrm{Fig}$. 38 was effective in preventing smoke aerosols from plugging the downstream HEPA filter.

Figure 46 shows the test results of placing our rolling prefilter in the ventilation duct upstream of a HEPA filter after the composite crib was ignited in the fire test cell. We have platted the pressure drop across the prefilter and HEPA filter as a function of time from the start of the fire. Figure 46 also shows the tota? air flow through the ventilation duct.

The primary information obtained in this experiment is the effectiveness of the RPF to prevent the smoke aerosol from plugging the downstream HEPA filter. The oscillations in the pressure drop across the prefilter up to $2500 \mathrm{~s}$ are due to repeated cycles of filter plugging and filter advancement. The pressure fluctuations across the prefilter are also somewhat reflected in the air flow rate. In order to assess the effectiveness of the prefilter to prevent the HEPA from clogging, the prefilter media was cut at $2500 \mathrm{~s}$ into the experiment. After this time, the pressure drop across the HEPA begin to increase dramatically, and the HEPA essentially clogged $w$ ith in another $400 \mathrm{~s}$. This test shows the rolling prefilter is very effective in protecting the downstream HEPA from the smoke aerosol. 


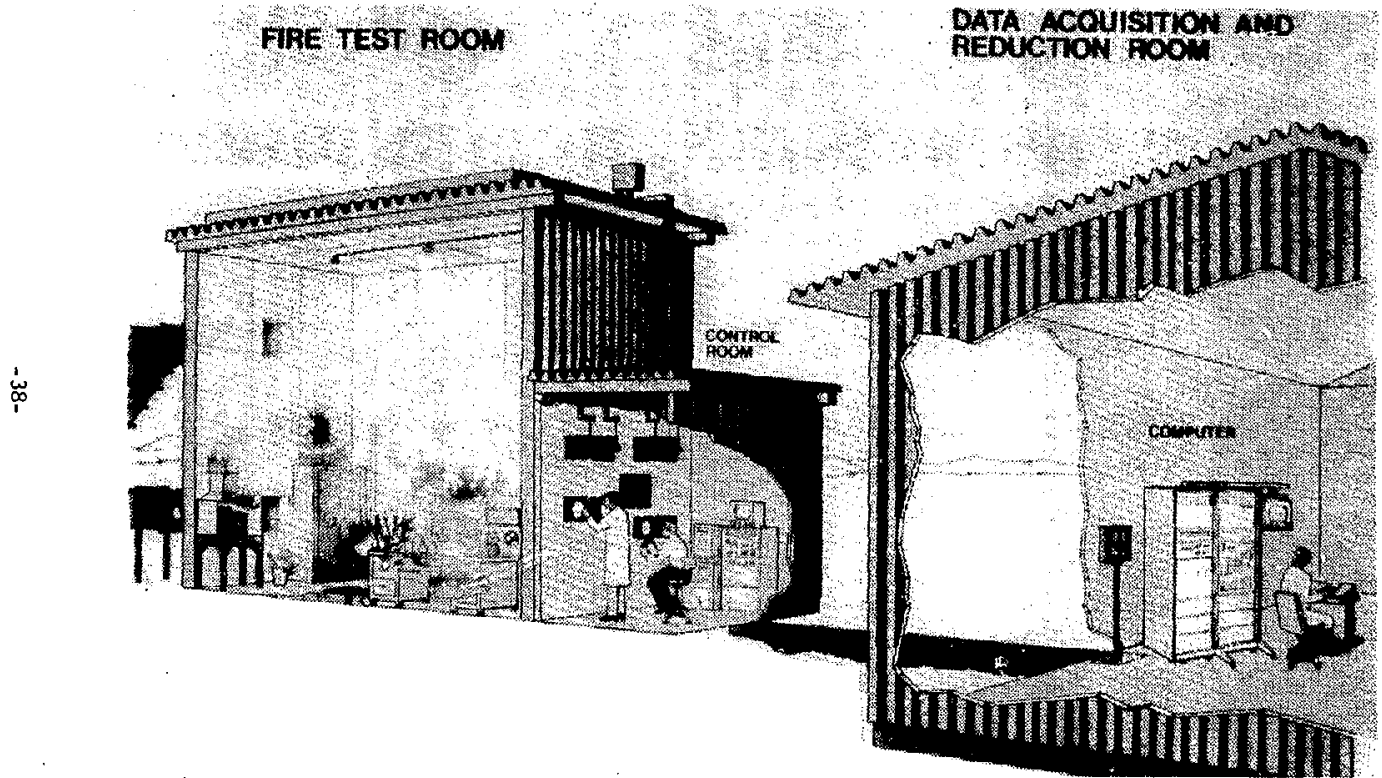

Figure 44. Drawing of fire test facility used to evaluate rolling prefilter under fire conditions. 


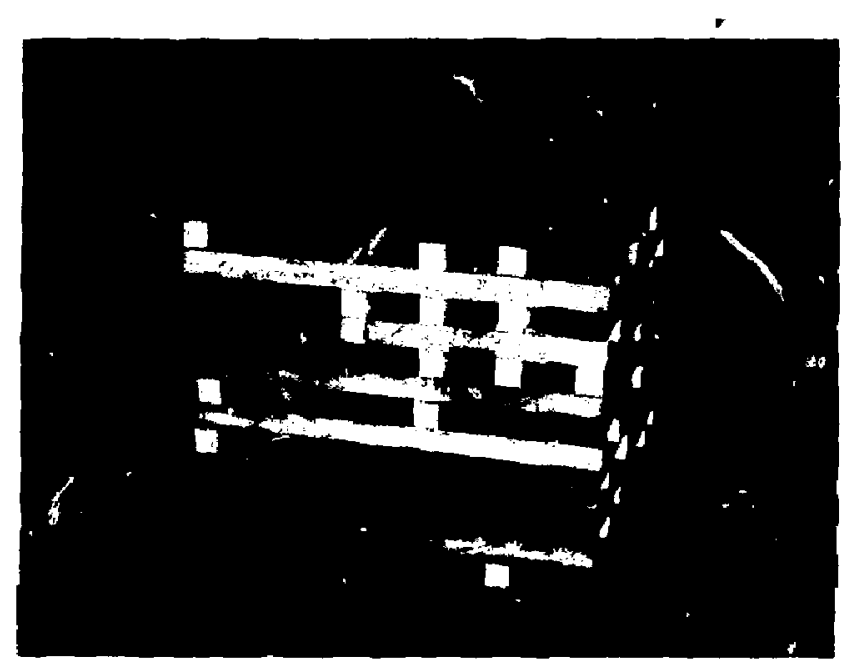

Figure 45. Photograph of composite crib used as the fuel source

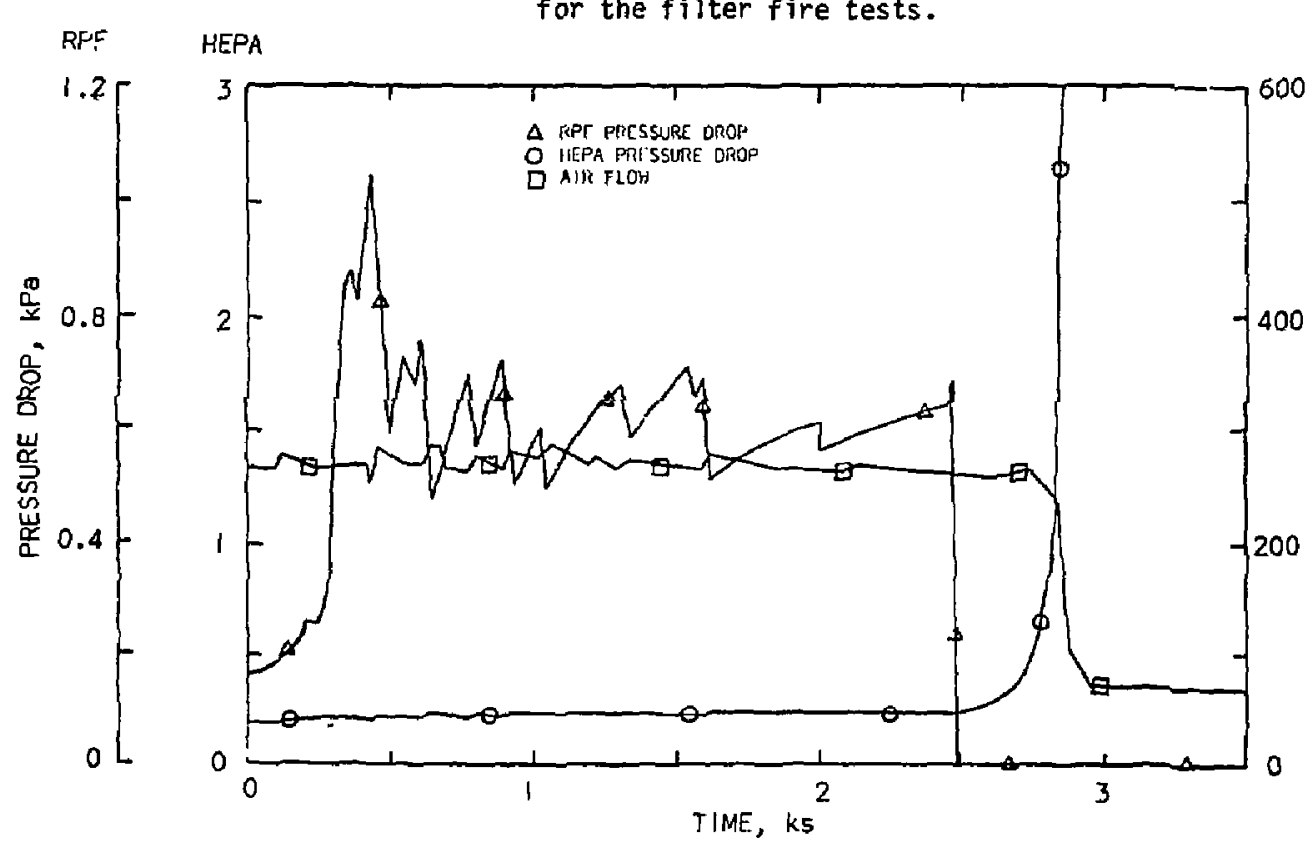

Figure 46. Test results illustrating the effectiveness of the roling prefilter in protecting the downstream HEPA filter from smoke aerosols. 


\section{Evaluation of Recirculating Electric.Air Filters at Savannah River}

\section{A. Introduction}

We selected the 236 40 fuel fabrication facility in Building 235- $F$ at the Savannah River Plant for demonstrating our electrofibrous filter technology for several reasons. First, this facility has an extremely severe particulate problem that cannot be controlled by conventional nuclear air cleaning designs. The severity of the particulate problem is not only due to the relatively high concentration of suspended particles, but also to the extrememely high radioactivity of the particles, Based on a maximum $10 \mathrm{~g}$ loading on a HEPA filter operating at $80 \mathrm{cfm}$ over one month, we estimate the maximum average particulate concentration to be $0.2 \mathrm{mg} / \mathrm{m}^{3}$. Of course the instantareous concentration may be much higher than this average.

The second reason for selecting this installation site is the small size of the suspended particles. Measurements of the particle size distribution with a Coulter Counter indicated that most of the particles fell below the instrument detectability of $0.5 \mu \mathrm{m}$ diameter. (y) The small size of the radioactive particles presents a major challenge to any proposed air cleaning technology. The use of electrofibrous filters is ideally suited for this installation since they have a dramatic improvement in collection efficiency for particles below $1.0 \mu \mathrm{m}$ when compared to conventionial fibrous

The third reason for selecting the fuel fabrication site is the substantial savings in operational cost that would result from a major reduction in the particulate concentration. We estimate that a theoretical reduction of 100\% of the suspended particles will result in an annual savings of $\$ 245,000$ in operational costs, not including the burial cost of high level radioactive waste. A realistic reduction of 70\% of the suspended partictes. will yield an annual savings of $\$ 772,000$. Any one of the three reasons would provide sufficient justification for selecting the fuel fabrication facility for demonstrating the electrofibrous filter technology. In combination, these three reasonj make the Savannah River evaluation our most important demonstration to date.

The Savannah River evaluation is also important because the prototype design offers an alternative approach to nuclear air cleaning. Our other prototypes are designed for existing ventilation systems as prefilters to HEPA filters. The installation of these prototypes of ten involve an expensive retrofit to existing ventilations systems and have constraints placed on the design since the electrofibrous prefilters become components of existing ventilation systems. In contrast, the prototype design for the Savannah River evaluation is a self-contained unit that is independent of the existing ventilation system. As such, it avoids the costly retrofit and severe design constraints when installed in ventilation systems. The prototype design is a self-contained unit that recirculates contaminated air from a confined space through an electrofibrous filter and blower and then exhausts into the same conf ined space. 


\section{B. Description of the Facility Operation and the Particulate Problem}

The fuel fabrication facility in Building 235-F consists of nine interconnected cells in which $238 \mathrm{PuO}_{2}$ powder is formed into fuel pellets that serve as a heat source for thermoelectric generators. The process of converting the powder into the fin ished pellet is primarily done remotely using mechanical manipulators with a small number of operations performed using lead lined gloves. There are also two electric motor hoists in cell 1 and one in cell 5 for transporting material.

The operation begins with bagging in the raw $\mathrm{PuO}_{2}$ powder into a wing cabinet behind cell 1. This operation is performed manually using lead lined gloves. The powder is then transferred to cell 1 where it is poured into a container which is inserted into an axygen exchange furnace. Here the naturally occurring isotopes of $1 \%_{0}$ and 180 are exchanged with 160 to reduce the neutron emission from $\mathrm{PuO}_{2}$, thereby making the product safer to handle. Following the oxygen exchange, the $\mathrm{PuO}_{2}$ powder is poured into another container and transferred to cell 2 where the powder size is reduced in a ball mill. Since the powder being ball milled is enclosed in a container, suspended particulates are not generated in cell 2. The high concentration of suspended particulates in cell 2 results from the passage of particles through the door connecting cells 1 and 2 . After the ball milling operation, the contained powder is transferred back into cell $]$. Here, the container is opened and the $f$ ine powder poured into another furnace that sinters the powder into larger sized granules.

The PuO granules are then poured into another container which is passed through cells 2 and 3 into cell 4 . There are no operations in cell 3 . It functions as a buffer zone between the powder operations in cells 1 and 2 and the pellet operations in the remaining line. The PuOn granules are poured into a hot press in cell 4 and made into a monolithic pellet. This pellet is then transferred to cell 5 where it is placed in a heat treat furnace and exposed to high temperature, thereby forming it into its final state. Following the heat treatment, the pellet is transferred back to cell 4 where the pellet is out gassed under vacuum. All of the operations in cells t-5 are conducted in a purified argon atmosphere free from water vapor. The pellet is then transferred through cell 5 into cell 6 . Here the pellet is placed inside an irridium cup and sealed by welding. The operations in cell 6 are conducted in a helium atmosphere. The sealed $\mathrm{PuO}_{2}$ is then decontaminated in cells 7 and 8 and finally placed in a shipping container and welded shut in cell 9. Since the $\mathrm{PuO}_{2}$ is well sealed inside the irridium container, cells 7-9 are maintained in an air environment.

The entire operation in the $n$ ine cells can be grouped into three major divisions according to the form of $\mathrm{PuO}_{2}$ and the level of suspended particulates. In cells 1 and 2 the $\mathrm{PuO}_{2}$ is a fine powder and the celis have a high concentration of suspended particulates. The particles become suspended in the pouring and other powder handling operations. Celis 3-5 handle the $\mathrm{PuO}_{2}$ in the form of large granules or a monolithic pellet and results in a rellatively low concentration of suspended particles. Cells $6-9$ have a negligible concentration of suspended particles since the $\mathrm{PuO}_{2}$ is sealed inside welded containers. 
The operational problems created by the suspended particles can likewise be categorized according to the three divisions with cells 1 and 2 representing the worst case, cells 3-5 an intermediate case and celis 6-9 having no operationai problems created by these suspended particles. Among the operational problems created by the radioactive dust particles are premature failure of the mechanical manipulators, electric hoists, gas lines, thermocouple leads, lead lined gloves, and vacuum gauges. The failure in each of these cases is due to the intense radioactivity destroying the component directly or weakening its strength sufficiently to cause a premature mechanical failure. In addition to these direct operational problems, the suspended particles also require a frequent change out of HEPA filters.

of all of the operational problems created by the radioactive particulates, the most serious is the premature failure of the mechanical manipulators. A mechanical manipulator consists of three basic components: a master outside of the cell powered by an electric motor and controlled by an operator, a slave inside of the cell that is controlled by the master, and a through tube that connects the master to the slave. The suspended particles in the cell cause a mechanical tailure of the slave which must be discarded as high level radioactive waste because it cannot be decontaminated. The conventional solution to protecting the slave from a dusty environment is to cover the slave with a boot. Unfortunately, the radiation from the particle deposits destroys the boot material in less than two weeks of operation. The disintegrated boot then interfers with the slave movements and creates a worse problem than operating the slave without a boot.

A common failure mode in the mechanical manipulator is the release of the cable straps in the turn buckle. The turn buckle is used to connect two cable straps together in a similar fashion as a link on a chain. Each end of the cable strap is turned over itself and clamped to form a loop. A link between the two cable loops then connects the two cables together. Radicactive particles deposit in the turn buckle and weaken its hold on the cable sufficiently to pull the cable strap out of its loop. Another failure mode is the increased friction on components that slide over each other. Excessive force is then required to move the manipulator which leads to premature failure.

\section{Component Replacement Costs}

When a manipulator fails, arrangements are generally made to have it replaced during the following weekend or another convenient weekend. In the meantime, the adjacent manipulators that are still functioning take-up the increased workload. Replacing a broken manipulator is a major operat ion and requires approximately three men work ing 32 hours each for a total of 96 hours. At $\$ 40$ per hour, this represents a labor cost of $\$ 3,840$. This is the labor cost for replacing manipulators in cells $7-5$. Since the contamination level in cells $6-9$ is cons iderably lower, the labor costs for replacing the manipulators will also be lower. We est imate it takes approximately 60 hours or $\$ 2,400$ to replace a manipulator in cells 6-9. In all cases, the fuel fabrication facility is shut down while the manipulator is replaced. The change out consists of building a tent around the defective manipulator, disconnecting the master from the through tube, and pulling out the defective slave. The through tube is then decontamined, a new slave inserted through the through tube and the master reconnected to the slave. The broken slave is then inserted in a PVC pipe and placed in a high level waste container about the size of a coffin. The cost for the slave is $\$ 9,000$ and the waste container, 
\$1,000. After the through tube is decontaminated six times it must also be replaced. Its cost is $\$ 9,000-\$ 12,000$, depending on the supply. The master also fails after 2-8 months of use, but since it is not contaminated it can be repaired and reused. It has a number of failure modes involving electrical and mechanical components.

We have tabulated the frequency of the manipulator change out and the associated costs for all of the cells in the $P_{u 0}$ fuel fabrication facility. Tabie 1 shows the manipulator replacement costs for all of the cells grouped together according to the concentration of suspended particles.

Table 1. Manipulator Replacement Costs

\begin{tabular}{|c|c|c|c|c|c|c|c|}
\hline $\begin{array}{l}\text { Part icle } \\
\text { Concentration }\end{array}$ & $\begin{array}{l}\text { Number } \\
\text { Cell }\end{array}$ & $\begin{array}{l}\text { of } \\
\text { Manipulator }\end{array}$ & Frequency & $\begin{array}{l}\text { lacemen } \\
\text { Labor } \\
\text { Cost }\end{array}$ & $\begin{array}{l}t \\
\text { Equipment } \\
\text { Cast }\end{array}$ & $\begin{array}{l}\text { Number } \frac{\text { Annua }}{\text { of }} \\
\text { Replacements }\end{array}$ & 1 \\
\hline high & 1,2 & 7 & 10.5 months & 3,840 & $\$ 10,000$ & 8 & $\$ 124,720$ \\
\hline med iun & $3-5$ & 8 & 32 months & 3,840 & $\$ 10,000$ & 3 & $\$ 46,770$ \\
\hline $10 \mathrm{~N}$ & $\underline{6-9}$ & 9 & 9 years & 2,400 & $\$ 10,000$ & 1 & $\$ 14,150$ \\
\hline TOTAL & $1-9$ & 24 & & & & 12 & $\$ 185,640$ \\
\hline
\end{tabular}

The equipment cost in Table 1 represents $\$ 9,000$ for the slave and $\$ 1,000$ for the waste container. We have also included $\$ 10,500$ in the annual cost for replacing the through tube at every sixth manipulator replacement. The frequency of the maniputator replacement in Table 1 represents approximate values and depends on a large number of variables including the hours of service, improper operator use and the number of manipulators out of service. However, the total annual number of manipulator replacements and the total annual costs are quite accurate. From June 1978 to September 1982 there have been 53 manipulators replaced. This averages 12.5 manipulator changes per year and agrees well with our estimate. During the same period of 4.25 years, there were 8 through tubes replaced. The ratio of manipulator replacement to through tube replacement indicates that the through tube was replaced after every 6.6 cleanings. Again, our estimates of the number of times the through tube is cleaned before it must be replaced agrees well with the actual data. We should also point out that the total cost for replacing 12 manipulators per year does not include the handling and burial of the 12 coff in sized boxes of high level radioactive waste.

Reducing the level of suspended particles would significantly decrease the annual cost of \$185,640 for replacing the mechanical manipulators. If the particulates were reduced $100 \%$, then a 11 of the manipulators would have a similar replacement frequency and cost as observed in cells 6-9. We calculate that 2.7 manipulators would be changed each year at a cost of $\$ 38,205$ thereby saving $\$ 147,435$. If the particulates were reduced by $70 \%$ the annual savings would still be $\$ 103,204$, a very significant amount.

A sinliar analysis can also be performed for the other items that must be replaced due to premature failure by the suspended particles. Tables 2 and 4 show the replacement frequency and cost for replacing the lead lined gloves, the electric hoists and the HEPA filters. 
Table 2. Gloye Replacement Casts

Replacement

Annual

\begin{tabular}{|c|c|c|c|c|c|c|c|}
\hline $\begin{array}{l}\text { Particle } \\
\text { Concentration }\end{array}$ & Cell & $\begin{array}{l}\text { Number of } \\
\text { Glove. Pairs }\end{array}$ & Frequency & $\begin{array}{l}\text { Labor } \\
\text { Eost }\end{array}$ & $\begin{array}{l}\text { Glove } \\
\text { Cost }\end{array}$ & $\begin{array}{l}\text { Number of } \\
\text { Glove pairs }\end{array}$ & Cost \\
\hline high & 1,2 & 9 & I week & $\$ 20$ & $\$ 108$ & 468 & 959,904 \\
\hline medium & $3-5$ & 6.5 & 2 weeks & $\$ 20$ & $\$ 108$ & 169 & $\$ 21,632$ \\
\hline low & 6-9 & 7.5 & 12 months & $\$ 20$ & $\$ 103$ & 7.5 & $\$ \quad 923$ \\
\hline TOTAL & $1-9$ & 23 & & & & 644.5 & $\$ 82,459$ \\
\hline
\end{tabular}

The failure mode of the gloves is a disintegration of the butyl rubber. As a safety measure the gloves are replaced on a preventative maintenance schedule rather than at the failure point.

Fábie 3. Ho ist Reglacement Costs

Replacement

Annual

Partic le

Number

Labor Hoist Number

Cancentration Ce ll

of Hoists Frequency

Cost Cost of Hoists

Cost

high

1,2

2

2 months

$\$ 320 \$ 460$

12

$\$ 9,360$

medium

$3-5$

5 months

$\$ 320$

$\$ 460$

2.4

$\$ 1,872$

low

$\underline{6-9} \quad \underline{0}$

2 years

$\$ 160 \$ 460$

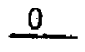

$\underline{\$ \quad 0}$

TOTAL

1-9 3

14.4

$\$ 11,232$

The failure mode of the electric hoists is motor failure when the $\mathrm{PuO}_{2}$ powqer penetrates the electric motor. The hoist chain also becomes britt and breaks due to the particle deposits.

Table 4. HEPA Replacement Costs

Replacement

Annual

Particie

Number

Labor Filter Number

Concentration Cell

Of. HEPAS

high

$1,2 \quad 2$

Frequency

Cost Cost

of HEPAS

$\underline{\text { Cost }}$

medium

3-5 $\quad 3$

1 month $\$ 160$

$\$ 73$

24

$\$ 5,592$

low

$\underline{6-9} \quad \underline{4}$

3 months $\$ 160$

$\$ 73$

12

$\$ 2,796$

TOTAL

1-9

18 months $\$ 80$

$\$ 73$

2.7

$\$ \quad 413$

9

$38.7 \$ 8.801$ 
The frequency of HEPA filter replacement is control led by a maintenance schedule to avoid having the used filters classified as high level radioactive waste. Savannah River classiffes any item having more then 10 grams of $23 \mathrm{puO}_{2}$ as high level radioactive waste.

The replacement costs for the vacuum and pressure hoses, thermocouple leads, and vacuum gauges can also be analyzed in a similar fashion. We have not done this since their total cost is very small compared to the items tabulated in Tables 1-4.

A summary of total anmual cost for replacing the four items tabulated in Tabies $1-4$ is given in Table 5 . We have also tabulated the total annual cost for replacing these items for the case of $100 \%$ particulate removal. These calculations are made by assuming the replacement frequency for the items in cells $1-5$ are the same as in cells $6-9$.

Table 5. Total Annual Replacement Costs

\begin{tabular}{|c|c|c|c|}
\hline Item & Present cost & $\begin{array}{l}\text { Cost with } 100 \% \\
\text { Particulate Control }\end{array}$ & $\begin{array}{l}\text { Potentia } \\
\text { Savings }\end{array}$ \\
\hline Manipulators & $\$ 185,640$ & $\$ 38,205$ & $\$ 147,435$ \\
\hline Gloves & $\$ 82,459$ & $\$ 2,829$ & $\$ 79,630$ \\
\hline Ho ists & $\$ 11,232$ & $\$ 930$ & $\$ 10,302$ \\
\hline HEPA Filter & $\$ 8 ; 801$ & $\$ 918$ & \$. 7,983 \\
\hline TOTAL & $\$ 288,132$ & $\$ 42,882$ & $\$ 245,250$ \\
\hline
\end{tabular}

We see that substantial cost savings can be obtained by controling the particulate problem.

\section{Prototype Besign}

The method used for controlling the particulate problem in the fuel fabrication facility was largely determined by the operating conditions of the facility. Each of the cells has a ventilation system to remove suspended particles For example, the ventilation system in cell 1 removes a volume of contaminated argon equal to the cell volume every $7-1 / 2$ minutes $(80 \mathrm{~cm}$ exhausted from a 600 cubic foot celi). Although the ventilation system would normally be considered adequate for most applications, the high radiaactivity and small size of the suspended particles create major operational problems. In most nuclear facilities the major operational problem due to high particulate concentrations is the frequent replacement of HEPA filters. We have addressed this problem in our previous field eyajuations by adding a low maintenance prefilter upstrean of the HEPA filter. $(1,5,10)$ However, such a solution would not be effective in this fuel fabrication facility since frequent HEPA filter replacement is only a minor problem. An ideal solution would be to increase the existing ventilation rate. Unfortunately, this solution would very likely cost far more than the existing operating costs associated with the high particulate concentrations. 
After examining various methods for increasing the ventilation rate, we concluded that a recirculating air cleaner installed inside the cell would be the most cost effective method. The ideal recirculating a ir cleaner should have a high efficiency for the very small particle sizes and be able to clean large volumes of air. The electrofibrous filter meets these requirements very weli. Although electrostatic precipitators would also be effective, they would have serious maintenance problems with the corona wires breaking due to radiation damage. When the additional requirement of particle recovery is added to the selection of an air cleaner, the electrofibrous filter is the obvious choice. We have demonstrated the recovery of over $98 \%$ of plytionium salts in a previous field evaluation of the electrofibrous filter. (10)

However, in recovering $238 \mathrm{PuO}_{2}$ powder from the electrofibrous filter we have to be careful not to contaminate the recoverea powder with traces of the filter medium. Since the process of removing the particle deposits will also remove a trace amount of media fibers, one of the criteria for filter medium selection will be the compatability of the filter medium with the repracessing operation. This problem will be addressed after reaching our primary objective of minimizing the particulate concentration in the cell.

The design of the recirculating electrofibrous air filter was obtained by optimizing the following criteria: maximum air flow, maximum dust holding capacity, maximum filter efficiency, maximum dust recovery, minimum ma intenance, minimum size, simple installation and removal, insensitive to rough handing, minimum cost, and no high voltage hazard. These criter ia had to be optimized while meeting the constraints of the cell, the primary constraint being the size of the entry/exit port. In order for the filter to be readily inserted inta and removed from the cell, the filter had to fit through a 14" diameter port.

The criteria of maximum air flow and maximum filter efficiency determine the performance of the recirculating electrofibrous air filter in reducing the particulate level in the cell. These two criteria are inversely related since increasing the fitter efficiency decreases the blower air flow due to the increasing filter pressure drop. We trave optimized these two criteria by selecting a blower that $f$ its through the 14 inch port and pulls the maximum a ir flow against the resistance of a medium efficiency $f i l t e r$. By electrifying the filter media we then convert the medium efficiency filter to a high efficiency filter. Increasing the surface area of the filter also decreases the flow resistance and increases the filter efficiency. The blower-filter combination that we selected pulls $200 \mathrm{cfm}$ and has $98 \%$ efficiency for submicron aerosols. The blower is a high pressure centrifuga? blower (IGL model $7-1 / 2 \mathrm{P}$ ) and the fijter is a $/ / 2$ in. thick glass fiber matt (Johns Manville, AF-18). This combination will increase the ventilation rate in cell 1 from $80 \mathrm{cfm}$ to $280 \mathrm{cfm}$.

Assuming a linear relationsh ip between particulate removal and ventilation rate, we can expect a 71\% reduction in the particulate level in cell 1 . ATso assuming a linear relationship between the particulate concentration and cell equipment failure, we can expect a $71 \%$ reduction in replacement costs, Based on these assumptions we predict that installing recirculating electrofibrous air filters in cells $1,2,4$ and 5 will yield an annual savings of $\$ 172,000$ in equipment replacement costs. 
The remaining criteria are intended to minimize the operational problems and maximize the recovery of $\mathrm{PuO}_{2}$. The maximum dust holding capacity and maximum dust recovery require a large amount of filter media which will increase the size of the filter unit. Moreover, in order to recover the dust deposits, the filter surface that collects the particles must be smooth, thereby eliminating the option of convoluting the media to increase its area while maintaining an overall small size. We have optimized the criteria of dust holding capacity, small size and dust recovery with a cylindrical filter cartridge 1 foot diameter and 3 feet long. By having the electric motor, high voltage power supply and other electrical components built inside the filter cartridge, the size nf the filter will be minimized. Filter maintenance will also be minimized by preventing the dust from contaminat ing the electric motor and other electrical components.

The design criteria were used to fabricate the prototype filter shown in Fig. 47. It is a completely self-contained unit and requires a standard $110 \mathrm{~V}$ input for its operation. The cylindrical filter cartridge slips over the filter housing and blower with the aid of a handle mounted on top of the filter cartridge. Two clamps, not shown in Fig. 47 , secure the filter cartridge to the blower housing and provide an electrical ground to the outer electrode. Figure 48 shows the filter cartridge removed thereby expasing a

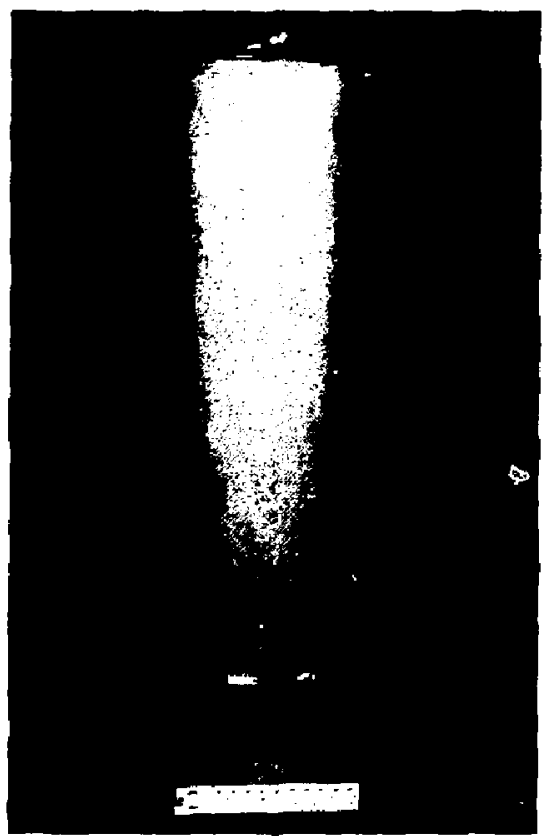

Figure 47. Recirculating electrofibrous air filter

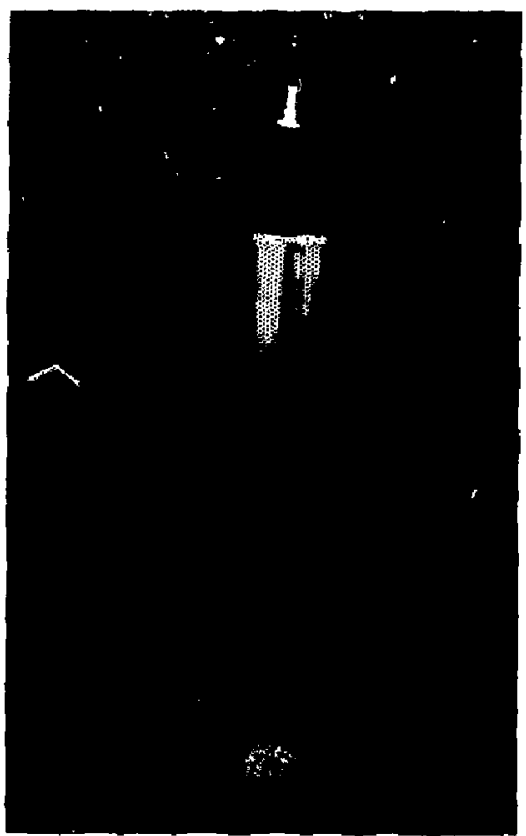

Figure 48. Recirculating electrofibrous air filter with filter cartridge removed 
grounded cylindrical screen. It protects the electrical components from physical damage and provides a guide for inserting the filter cartridge. High voltage is applied to the interior screen of the filter cartridge by means of a piano wire fastened to the high voltage lead that extends from the top of the cylindrical screen. The high voltage output poses no electrical hazard since this power supply cannot exced $70 \mu \mathrm{A}$ at $10 \mathrm{kV}$. One can physically touch the piano wire and not feel the electrical output.

The cast iron blower and electric motor serve as the base of the filter unit and prevent the unit from tipping over if it is bumped or roughly handled. A closeup of the blower and base of the filter unit is shown in Fig. 49. Note the impeller inside the cast iran housing of this centrifugal blower. We have modified the blower configuration so that filtered air wil? be pulled past the electric motor into the blower housing and exhausted through the port shown in Fig. 49. This madification significantly reduces the size of the filter, lowers the center of gravity for increaed stability, and places the electric motor inside the filter cartrige, thereby prevent ing motor failure due to dust particles. Since all of the filtered air will be pulted across the electric motor, there will be adequate cooling of the motor.

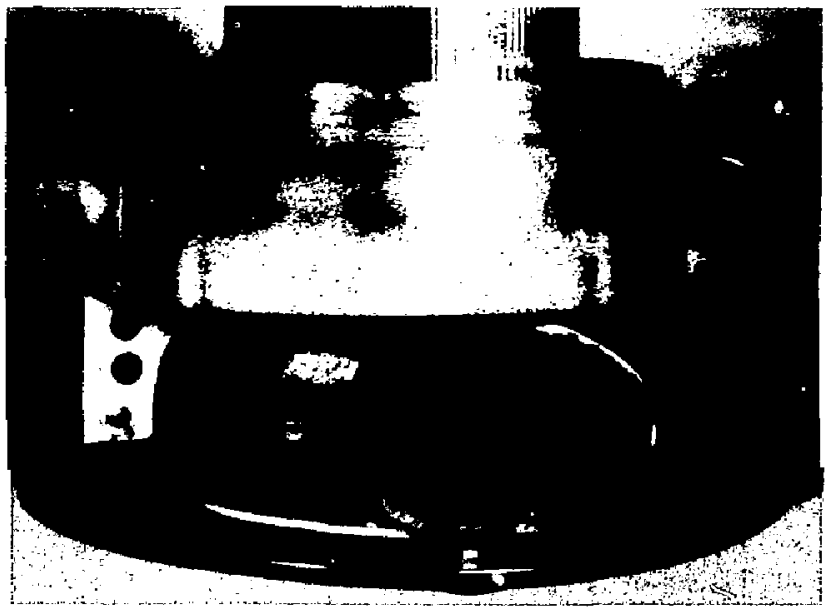

Figure 49. Base of the filter consisting of an electric driven air blower and the supporting flange for the filter cartridge.

Figures 50 and 51 show the filter housing after the grounded screen is removed. The cylindrical screen is easily removed from the housing by rentoving a retaining nut, thus providing access to the electrical components. The rectangular box in Fig. 50 is the solid state high voltage power supply. With a 15 volt D.C. input, it provides $10 \mathrm{kV}$ output to the high voltage lead 
at the top of the unit. Figure 53 shows the opposite side of the filter housing. The rectangular box in this figure is a solid state transformer that converts the $110 \vee A . C$. into $15 \vee D . C$. for supplying the input voltage to the high voltage power supply. A string of three resistors in series connect from the high voltage lead on top to a logic circuit that senses if there is an over load. If there is an excessive drain on the power supply due to some failure mode, a light on the electrical contral box will turn on. The electric control box, shown in Fig. 52, has two toggle switches to operate the filter unit, a power switch to activate the bJower and a separate high voltage switch to provide high voltage to the filter cartridge.

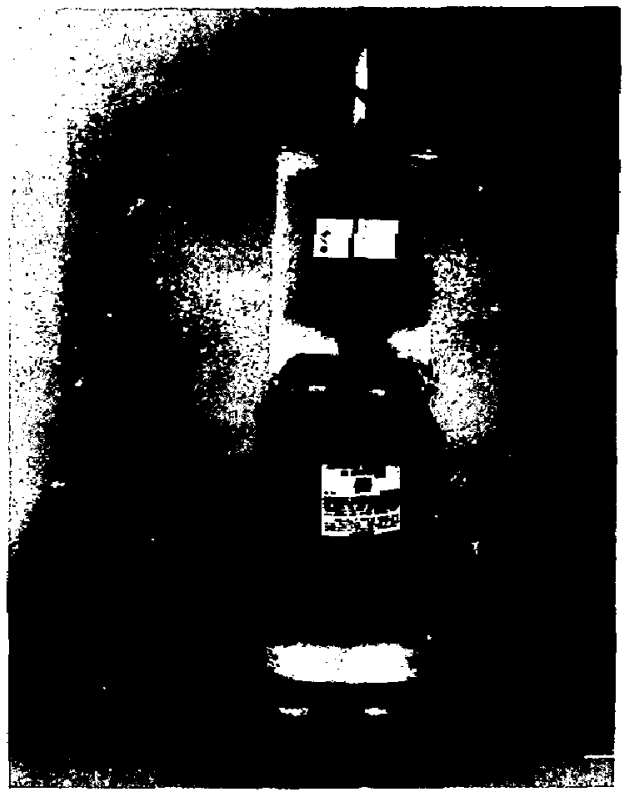

Figure 50. Fitter housing with the protective screen removed exposing the high voltage power supply and electric motor.

The electrofibrous recirculating air filter shown in Figs. 47-52 is designed for easy installation and operation. The blower assembly, Fig. 49, and filter cartridge are bagged into the cell as separate units. Once in the cell, the filter cartridge is inserted over the housing and an electric cord plugged into the filter and the cell outlet. The blower and high voltage are then turned on with the respective toggle switches.

\section{E. Laboratory. Evaluation}

The electrofibrous recirculating air filter was evaluted in an LLNL test cell under controlled laboratory conditions designed to simulate actual operation in a cell of the 238 puo fuel fabrication facility in the Savannah River Plant. The test cell has a volume of 354 cubic feet $(5.5$ feet 
spaces or to air flow patterns, the performance of the recirculating air filter will decrease accordingly. A full analys is of these additional characteristics is required to evaluate the performance of our prototype f fiters. We hope to complete this analys is during the course of our field evaluation.

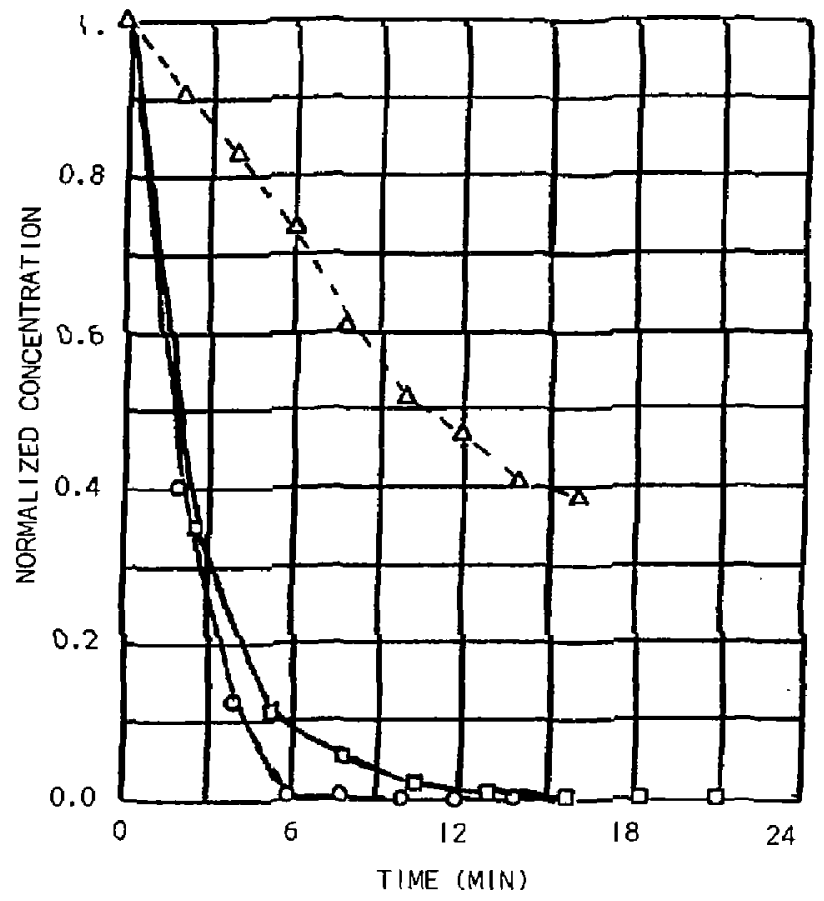

Figure 53. Decay of D0S aerosol concentration in LLNL test cell with and without recirculating electrofibrous air filter. 


\section{F. Field Evaluation in $23 \mathrm{PuO}_{2} \mathrm{Cell}$}

We shipped the prototype recirculating air filter, shown in Fig. 47 , to the Savannah River Plant for installation in cell 1 of building 235-F where 230 pu 0 powaer is formed into pellets for use as heat sources. However, before the filter could be installed in the cell an anaysis of the safety features and operating performance was required. The results of the safety analysis indicated that the filter unit could be operated safely inside the cell after several electrical connections were upgraded. The paint on the blower and other components of the filter housing also had to be relloved to prevent contamination of the $\mathrm{PuO}_{2}$ powder.

The primary concern with the operating performance was the residual amount of $\mathrm{PuO}_{2}$ remaining on the filter media prior to its disposal. Since any item containing more than 10 grams of $238 \mathrm{PuO}_{2}$ must be treated as high level radioactive waste, the management of this facility wanted assurance that the filter would have less than 10 grams of residual $\mathrm{PuO}_{2}$ remaining on the filter media after cleaning. Our estimates of dust holding capacity and resioual oust after cleaning greatly exceed this 19 gram limit. In our previous evaluation of an electrofibrous prefilter (10) we could load up to 10 grams of Pu salts on a filter area of $0.4 \mathrm{ft}^{2}$. After cleaning off the dust deposits, the filter had a residual deposit of two grams. Assuming similar values of particle deposits for our recirculating air filter, we calculate a maximum loaaing of 235 grams and a residual deposit of 47 granis after cleaning. Based on a maximum $10 \mathrm{gram}$ lintit trapped in a HEPA filter atter one month operation, we calculate that the recirculating air filter will have a maximum 235 grams after 9 months of operation. The filter can be cleaned by knocking off the dust deposits (recovering 188 grams of powder) and reused for arother oust loading interval. Even if the filter becomes

inoperative after only one cleaning due to raaiation damage, it has eliminated far more high level waste than it has produced. During the nine month period the recirculating air tilter would eliminate the replacenent of six manipulators, all of which would be handled as high level waste.

If the disposal of the filter unit as a high level waste is to be avoided at all cost, then the filter cartridge can be replaced on a rautine maintenance schecule as is oone for the HEPA filters. As suming a change out rate proportional to the volumetric flow rate, the cylindrical filter cartridge should be replaced every 7.5 weeks. The primary disauvantage of this approach is the elimination of any recovery of $\mathrm{PuO}_{2}$ powder. If the recovery of $\mathrm{PuO}_{2}$ powder is no longer a criterion then the size of the filter cartrioge can be reduced from a three foot length to one foot by pleating the filter medium. This option will be examined in greater detail following the initial evaluation of the prototype shown in Fig. 47.

Another problem that had to be addressed prior to installing the recirculating electrofjbrous a ir filter was the technique for measuring the concentration of suspended $\mathrm{PuO}_{2}$ particles. Since we calculate the maximum $\mathrm{PuO}_{2}$ concentration in the cell is $0.2 \mathrm{mg} / \mathrm{m}^{3}$, gravimetric measurements of the filter sample will not be sensitive enough. In our previus evaluation at the Rocky Flats Plant in Golden, Colarado, we had collected suspended $239 \mathrm{pu}$ salts on an absolute filter and then measureo the alpha counts. However, a similar procedure for the current evaluation would not be possible because the $23 \mathrm{pu}_{\mathrm{u}}$ has a much higher alpha emission than the $239 \mathrm{pu}$ and would exceed the capability of the alpha meter. 
A more promising technique for measuring the $238 \mathrm{pu}_{2}$ concentration is to conduct a chemical analys is of $P u$ on the filter sample. Several sampies of the PuO will be taken in cell 1 and chemically analyzed to establish the particulate concentration. These measurements will provide a baseline concentation of $\mathrm{PuO}_{2}$ and will also establish the procedure to be used in determining the efficiency of the recirculating electrofibrous air filter. After the procedure for measuring $\mathrm{PuO}_{2}$ dust is established and several baseline measurements are made, the recirculating air filter shown in Fig. 47 will be installed in cell 1. Measurements of $\mathrm{PuO}_{2}$ concentration in the cell and in the blower exhaust will establish the efficiency of the filter unit in removing PuOz particles as well as the decrease in particle concentration in the cell.

The initial phas of the eva?uation will determine the filter performance at essentially zero dust loading after 1.5 weeks of operation. The filter unit will be removed from the cell to insure that less than 10 grams of $\mathrm{PuO}_{2}$ are trapped on the filter meida. Once the filter performance data are established, the filter design and mode of operation will be reviewed to determine the next $F$ ase of the evaluation. Although the primary objective of the evaluation is ciearly the reduction of the high maintenance cost due to the $\mathrm{PuO}_{2}$ dust, the secondary objective of recovering the $\mathrm{PuO}_{2}$ powder is not as clear. The problem stems from the desire to have less than 10 grams of Puon on the filter $k$ inen it is finally decommissioned and removed from the cel1. The filter unit shown in Fig. 47 was designed for maximum recovery of particle deposits by forming a heavy deposit of $\mathrm{PuO}_{2}$ powder on the fifter surface (about $235 \mathrm{grams}$ ). Although most of the deposit can be removed by mechanically tappinc the filter, the residual deposit of about 47 grams cannot be removed. There $i$, no question that the current design would significantly exceed the 10 gram 1 imit.

We foresee three possible solutions to the decomissioning problem. One solution is to remove the filter cartridge before it can accumulate 10 grams of $P_{u O n}$ as is done $w i$ th the existing HEPA fiters. This is the least satisfactory solution since no material could be recover $d$ and the frequent filter changes (every 1.5 weeks) would add appreciably to the maintenance costs. Another solution would be to operate the filter as designed for allowing recovery of $\mathrm{PuO}_{2}$ pawder and treating the filter when decommissioned as high level waste. The decommissioning of the filter cartrige as high level waste would certainly be offset by eliminating the replacement of six manipulators that would also be treated as high level waste. A compromise solution that would permit the filter to be operated for extended periods without maintenance and allow for recovery of PuOz powder is to redesign the cartridge so that it can be easily divided into a number of components, none of which would exceed the $10 \mathrm{gram} l$ imit. We believe this solution car be ach ieved with only minor changes to the filter cartridge shown in Fig. 47. 


\section{Summary}

Numerous studies have shown that applying an electric field across a fibrous filter medium will significantly improve its efficiency and dust holding capacity. The application of an electric field is accomplished by sandwiching high voltage screens between a fibrous medium. In this report, we described the design, fabrication and evaluation of four prototype

electrofibrous filters using these principles. These prototypes differ in the specific design depending on the intended application and method for changing the filter medium.

Two of the prototypes were designed for use in standard nuclear ventilation ducts as prefilters to HEPA filters. One of these prototypes is designed as a permanent filter unit in which the front electrade is mounted on sliders that permit easy replacement of the filter medium. The second prefilter design is a disposable filter that consists of a pleated electrified filter medium attached to a supporting frame. The electrodes are a wire cloth glued directly onto the filter medium. In this design the entire filter is discarded when the filter medium becones plugged. The disposable filter was evaluated at LLNL in the exhaust stream of a glove box in which barrels of uranium turnings and chips are burned. Preliminary tests show the electrofibrous prefilter is very effective in prolonging the HEPA filter 1ife.

Another prototype described in this report is the rolling electrofibrous prefilter. This prefilter was designed for use in applications requiring frequent replacement of HEPA filters or where HEPA filters must be protected in emergency conditions against smoke aerosols generated in fires. The filter medium in this prototype is automatically replaced by means of a continuous belt that advances the filter medium through the filter housing. Test results are presented in this report that demonstrate the roling prefilter can successfully protect HEPA filters fron plugging under the most severe smoke conditions.

The last prototype described in this report is a self-contained recirculating electrofibrous filter complete with its own blawer. This iilter unit is designed for use in cleaning confined spaces by recirculating the fixed votume of air through the filter. The filter element is a cylindrical cartridge consisting of a filter mediun sandwiched between two cylindrical electrodes. This alectrofibrous prefilter was shipped to Savamnah River to be installed in a hot cell where $P$ u-238 is manufactured into fuel cells for thermoelectric generators. Laboratory test results and a cost study show that four filter units will save $\$ 172,000$ per year due to maintenance costs due to the particulates. 


\section{Bibliography}

1. Bergman, W., Taylor, R. D., Miller, H. H., Biermann, A. H., Hebard, H. D., da Roza, R. A. and Lum, B. Y., "Enhanced filtration program at LLL - a progress report," Proc. 15th DOE Nuclear A ir Cleaning Conference. Boston, MA, August 7-10, 1978. CONF-7808T9, NTIS, Springfie d, VA. pp T058- 1098 (1979).

2. Nelson, G. 0., Bergman, W., Miller, H. H., Taylor, R. D., Richard, C. P., and Biermann, A. H., "Enhancedment of air filtration using lectric fields," Am Ind. Hyg. Assac: J., 39 pp. 472-479 (1978).

3. Bergman, W., Hebard, H., Taylor, R., and Lum, B., "Electrostat ic filters generated by electric fields," in Proceedings of the Second Wor ld Filtration Congress, September 18-20, 7979. London, England, Uptands Press Ltd., 1 katharine Street, Croydon CR9 ILB, England, unpaged, (1979).

4. Bergmen, W., Biermann, A. H., Hebard, H. D., Lum, B. Y. and Kuhl, W. D., "Electrostatic a fr filters generated by electric fields," Proc. of Fine Particle Society Meeting, : aryland (1980).

5. Bergman, H., Kuhl, W. D., Russell, H. L., Taylor, R. D., Mebard, H. D., Biermann, A. H., Alvares, N. J., Beason, D. G., and Lum, B. Y., "Electrofibrous prefilters for use in nuclear ventilation systems," 16th DOE Nuclear-A ir C. leaning Cciference, Oct. 20-23, 1980, San Diego, CA. CONF-801038, ATIS, SpringfieTd, VA, pp. 807-836 (1987).

6. Thomas, T. R., Exxon Nuclear Idaho.Company, Idaho Falls, Idaho, Private Communication, December (1981).

7. Alvares, N., Beasan, D., Gargman, W., Creighton, J., Ford, H. and Lipska, A., "Fire protection countermeasures for containment ventilation," Lawrence Livermore National Laboratory report UCID-18781, Livermore, California (1980).

8. Alvares, N. J., Beason, D. G. and Ford, H. W., "In-duct countermeasures for reducing fire-generated-smoke-derosol exposure to HEPA filters," Proc. 15th DOE Nuclear A ir. C Jeaning Conference. Boston, MA, August 7-10, 1978 , COMF-780819, NTIS, Springfield, VA PP 653-664 (1979).

9. Bickford, Dennis, Savannah River Plant, E. I. Du Pont de Nemours, Aiken, S.C. Private Communicaton, June (1981).

10. Bergman, W., Taylor, R. D., Hebard, H. D., Lum, B. Y., Kuhl, W. D., Boling, R. M. and Buttedah1, 0. I., Electrofibrous prefilters for use in the nuclear industry," Proc: Second $U$. S. DOE. Environmental Control Symposium, Reston, Virginia, pp. 334-37T (1980). 